\title{
Étude de la dynamique des masses d'eau du Lac de Pareloup par traçages
}

\author{
Marie-José Salençon* et Pierre Calmels ${ }^{* \star}$ \\ * Électricité de France, Direction des Études et Recherches, Département Environnement, \\ 6 Quai Watier, F-78401 Chatou Cedex, France. \\ ** Commissariat à l'Energie Atomique, C.E.N.G., Section d'Applications des Traceurs, \\ F-38041 Grenoble Cedex, France.
}

Résumé. - Un lac de barrage est le siège de circulations d'eau complexes liées à sa topographie, aux conditions météorologiques ainsi qu'à la gestion hydraulique des aménagements, pouvant perturber, de façon transitoire, son homogénéité horizontale.

Connaître ces circulations est important pour l'étude d'un écosystème de réservoir, car elles jouent un rôle fondamental sur le transport et la redistribution des nutriments à des échelles de temps de l'ordre de la journée.

Les circulations d'eau dans le lac de Pareloup ont été mises en évidence par l'injection de traceurs fluorescents (fluorescéine et rhodamine) qui ont marqué les eaux à différentes profondeurs, et lors de différentes situations hydrométéorologiques, en particulier: le suivi du panache de l'eau du lac de Bage injectée au fond de la retenue par pompage, le régime de circulation des eaux de part et d'autre de détroit de Charouzech, les échanges d'eau entre le bras du Rieutord et le corps principal de la retenue.

Mots-clés. - Circulations d'eau, fluorescéine, Pareloup, réservoir, rhodamine, traceur fluorescent, upwelling.

Abstract. - A dammed lake is the site of complex movement of water linked to topography and to meteorological conditions, as well as to the hydraulic management of the dam installations which can, for short periods of time, disturb its horizontal homogeneity. Knowledge of circulation patterns is important in studying a reservoir ecosystem, as they play a key role in transport and redistribution of nutrients on time scales on the order of one day.

Circulation patterns in Pareloup Lake were pointed up by injecting fluorescent tracers (fluorescein and rhodamine) which marked the waters at different depths, and under different hydrometeorological conditions, in particular: movement of the plume of water from the Bage pumped into the bottom of the reservoir, patterns of circulation of water on either side of the Charouzech strait, water exchanges between the arm of the Rieutord and the main body of the reservoir.

Key-words. - Circulation patterns, fluorescein, fluorescent tracer, Pareloup, reservoir, rhodamine, upwelling. 


\section{INTRODUCTION}

Dans le milieu naturel, une masse d'eau au repos (telle qu'un lac ou un réservoir) est en contact avec le milieu extérieur par sa surface, sur laquelle s'exerce la pression du vent, et par les entrées d'eau qui l'alimentent (rivières, pompages etc...). A ces sollicitations extérieures, cette masse d'eau répond par une mise en mouvement du fluide. Lorsque cette masse d'eau est stratifiée, des ondes internes se propagent le long des surfaces de fort gradient de densité qui constituent des surfaces de discontinuité. Dans un lac, c'est la thermocline qui joue ce rôle. Si les déplacements restent de faibles vitesse et amplitude, la masse d'eau s'adapte à cette perturbation et la structure thermique est maintenue. $\mathrm{Si}$ cette perturbation va au-delà des capacités de réponse propre de la masse d'eau, les mouvements oscillatoires dégénèrent en instabilités source de turbulence. La turbulence ainsi créée est utilisée pour un mélange local et la structure interne peut être détruite.

Ainsi, une masse d'eau apparemment calme comme un lac est le siège de circulations d'eau complexes liées à sa topographie, aux conditions météorologiques ainsi qu'à la gestion hydraulique des aménagements, pouvant perturber, de façon transitoire, son homogénéité horizontale.

Connaître ces circulations est important lorsque l'on étudie un écosystème de réservoir, car elles jouent un rôle fondamental sur le transport et la redistribution des nutriments à des échelles de temps de l'ordre de la journée.

Le lac de Pareloup, avec sa forme digitée qui présente des rétrécissements pouvant isoler certaines parties, est soumis, de plus, à une gestion hydraulique par pompage et turbinage alternatifs, qui créent des circulations particulières.

Ces différentes circulations ont été mises en évidence par l'injection de traceurs fluorescents (fluorescéine et rhodamine) qui ont marqué les eaux à différentes profondeurs, et lors de différentes situations hydrométéorologiques.

\section{TECHNIQUES DES MARQUAGES DE L'EAU D'UN LAC}

\section{II.1 Objectif des traçages}

Les techniques utilisées pour marquer l'eau d'un lac afin d'en suivre les circulations, sont dérivées des techniques mises en œuvre pour marquer les cours d'eau et les rejets en mer (Gilath et Gonfiantini, 1983).

Cependant, les phénomènes hydrodynamiques qui s'établissent dans un lac, directement liés à la géométrie des fonds et aux conditions météorologiques, nécessitent une méthodologie particulière.

Les mouvements des masses d'eau dans un lac sont généralement 
trop faibles pour être mesurés avec précision par les techniques hydrographiques usuelles.

Ils sont accessibles d'une manière relativement simple par la mesure de la migration d'une masse d'eau marquée avec un traceur radioactif ou chimique, décelable dans l'eau à très faible concentration.

Il est ainsi possible de recueillir des informations sur:

- la pénétration et la dispersion d'un apport dans un lac (cours d'eau, pompage...);

- les circulations sublacustres engendrées par le vent en présence ou non d'une stratification thermique.

A partir du marquage de l'eau qui pénètre dans le lac, il est possible de mesurer les vitesses de propagation du flux entrant, la répartition longitudinale, transversale et verticale de l'apport dans le lac et son taux de dilution à des dates précises.

Outre les circulations locales créées par les apports ou les soutirages, les circulations sublacustres sont principalement engendrées par le vent et les gradients de densité.

Des injections de traceur en des points judicieusement choisis, permettront d'individualiser ces circulations sur de grandes distances (plusieurs kilomètres), de déterminer les conditions de leur déclenchement (vitesse et direction du vent) et de mesurer les paramètres hydrauliques des courants qu'elles engendrent (direction, vitesses, périodes caractéristiques, coefficient de dispersion).

\section{II.2 Principe de la mesure des mouvements de l'eau}

Le déplacement d'une masse d'eau et la dispersion qui en résulte, peuvent être observés à partir du moment où cette masse d'eau a été individualisée par un traceur.

La méthode consiste donc à injecter une solution aqueuse de traceur dans l'apport ou en un point du lac, choisi pour sa représentativité (épilimnion - hypolimnion - détroit - etc...) puis à suivre l'évolution du panache d'eau marquée avec un dispositif de mesure in situ afin de pouvoir :

- décrire directement le déplacement et le volume occupé par l'eau marquée en fonction du temps;

- mesurer avec précision la concentration du traceur sur des échantillons d'eau prélevés sur la boucle de mesure in situ.

\section{II.2.1 Détermination des vitesses}

Des prélèvements réalisés en des points fixes situés sur l'axe des circulations étudiées, permettront l'acquisition de la fonction concentrationtemps de transfert de la masse d'eau marquée entre le point d'injection et le point de mesure.

Les paramètres temporels de transfert entre ces deux points, à savoir, le temps d'arrivée $\left(t_{a}\right)$, le temps modal $\left(t_{m}\right)$, le temps de séjour moyen (t) permettent le calcul des vitesses correspondantes sur la distance $\mathrm{L}$ d'observation. 


\section{II.2.2 Détermination des coefficients de dispersion}

Le calcul des coefficients de dispersion est fait à partir des valeurs des concentrations en traceur mesurées sur des profils transversaux et longitudinaux réalisés sur le nuage d'eau marqué à un temps moyen $t$ tel que la durée $\Delta t$ d'acquisition des profils peut être considérée comme négligeable par rapport au temps écoulé depuis l'origine $t_{0}$ du marquage.

L'interprétation des mesures de concentration peut être réalisée à l'aide d'un modèle numérique représentatif de la diffusion tridimensionnelle d'un fluide conservatif, à partir du rejet instantané et ponctuel effectué à une profondeur donnée dans un milieu horizontalement illimité, mais de profondeur finie (fig. 1).

II est montré que, sur un profil transversal réalisé à une distance $x$ constante, ou sur un profil longitudinal réalisé dans la direction du déplacement, l'expression de la concentration est de la forme:

$$
C=C_{m}(z, t) \cdot \exp \left(-\frac{A^{2}}{2 \sigma_{A}^{2}}\right)
$$

où $\mathrm{A}$ est égal à $\mathrm{x}$ ou $\mathrm{y}$ selon qu'il s'agit d'une exploration longitudinale ou transversale et $C_{m}(z, t)$ la concentration maximale sur une traversée dans le plan horizontal à la cote $z$ et au temps moyen $\mathrm{t}$.

Pour satisfaire l'équation de diffusion turbulente à une profondeur donnée, les variances $\sigma_{x}$ et $\sigma_{y}$ doivent vérifier les relations :

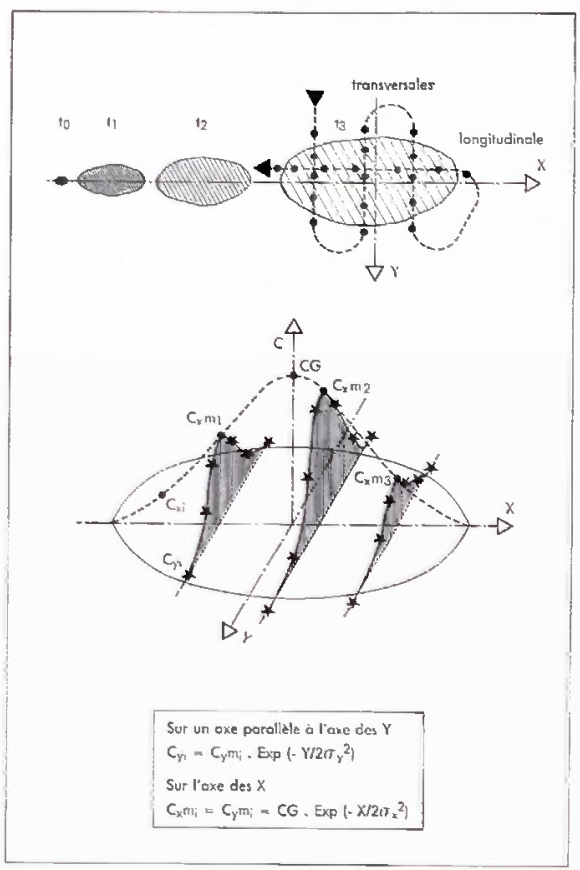

Fig. 1. - Détermination des coefficients de dispersion.

Fig. 1. - Determination of dispersion coefficients.

$$
\frac{d \sigma_{x}^{2}}{d t}=2 D_{x} \text { et } \frac{d \sigma_{y}^{2}}{d t}=2 D_{y}
$$

$\mathrm{D}_{\mathrm{x}}$ : incluant la diffusion turbulente et la dispersion longitudinale, cette dernière étant provoquée par les courants.

$D_{y}$ : étant le coefficient de diffusion turbulente transversale.

En pratique, les fonctions $C(x, y, t)$ obtenues à la cote $z$ sont ajustées, par la méthode des moindres carrés, à des gaussiennes qui permettent de déterminer les écarts-types $\sigma_{x}$ et $\sigma_{y}$ horizontaux en fonction du temps. 


\section{II.3 Techniques des marquages}

L'utilisation des traceurs pour marquer l'eau n'est pas récente. Cependant les technologies actuelles (le choix des produits, leurs mises en œuvre et les sensibilités de leurs mesures) ont permis de réduire d'un facteur 10 , voire 100 , les quantités de produit injectées avec, pour conséquences directes :

- un marquage de l'eau de meilleure qualité (pas de gradient de densité) ;

- une mise en œuvre allégée (pas de grandes masses de traceur à diluer dans de grands volumes d'eau).

\section{II.3.1 Sélection des traceurs}

Les produits utilisés pour marquer l'eau doivent répondre aux principaux critères suivants :
- être un bon marqueur de l'eau (sans en modifier la densité) ;

- ne pas interagir avec le milieu ni se dégrader trop rapidement;

- ne pas présenter de risques biologiques pour l'homme, la faune et la flore, ni esthétiques pour l'environnement ;

- avoir une teneur naturelle et une concentration minimale mesurable aussi faible que possible (de l'ordre de quelques $\mu \mathrm{g}^{-1} \mathrm{I}^{-1}$ ) permettant la mise en œuvre de quantités modestes ;

- être facilement détectables in situ à ces très faibles concentrations.

Des traceurs radioactifs tels que ${ }^{82} \mathrm{Br}$ et ${ }^{131}$ I répondent parfaitement à ces critères.

Toutefois, l'utilisation de fortes activités (de l'ordre de $370 \mathrm{GBq}$ ), la période de décroissance, le coût, l'approvisionnement, la mise en

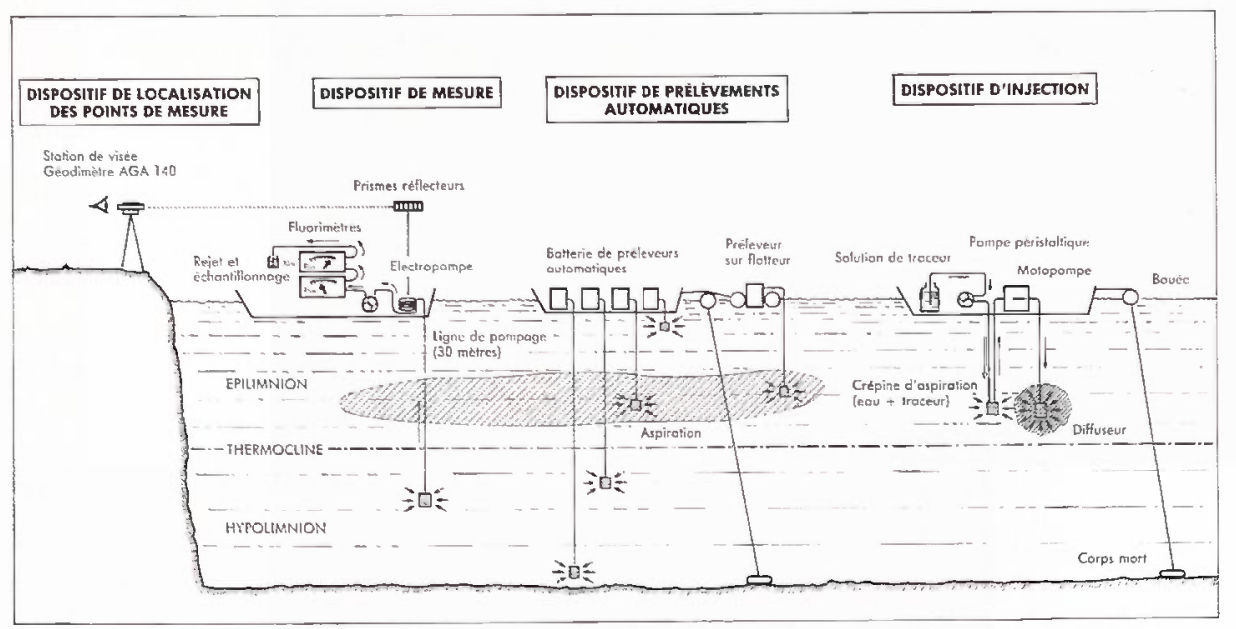

Fig. 2. - Dispositifs nécessaires à l'étude des circulations de l'eau dans un lac.

Fig. 2. - Materials needed to study circulation patterns in lake. 
$œ u v r e$ (containers très lourds - radioprotection) et les réglementations nationales et internationales d'utilisation des radio-isotopes limitant notablement leur usage, amènent les expérimentateurs à les remplacer par des produits fluorescents qui peuvent être mesurés in situ avec des sensibilités similaires (Gilath et Gonfiantini, 1983).

Ces produits sont:

- la Rhodamine WT en solution sodique et la Rhodamine B en solution acétique ;

- la fluorescéine et l'Amino G acid (en poudre).

Leurs caractéristiques de fluorescence étant différentes, ces produits sont aisément différentiables à l'analyse, même simultanément présents dans un même échantillon d'eau.

La concentration minimale mesurable, qui correspond à un doublement de la teneur naturelle, est de l'ordre de $2 \cdot 10^{-11} \mathrm{~kg} \cdot l^{-1}$ pour les rhodamines et de $2 \cdot 10^{-9} \mathrm{~kg} \cdot \cdot^{-1}$ pour les deux autres produits.

Afin de pouvoir procéder à des suivis simultanés en surface et au fond, nous avons choisi de marquer les eaux de l'épilimnion avec la fluorescéine que l'on peut détecter visuellement et celles de l'hypolimnion avec la rhodamine WT.

\section{II.3.2 Méthodologie des injections}

Deux modes d'injection sont utilisés pour marquer les masses d'eau dont on veut suivre la migration dans un lac. Ce sont l'injection continue, qui permet le marquage d'un flux d'eau en continu et l'injection instantanée qui individualise un volume d'eau bien localisé dans le temps et dans l'espace.

\subsubsection{L'injection continue}

Pour que le traceur soit réparti d'une manière homogène dans toute la section de l'apport, il est nécessaire que l'injection du traceur soit réalisée en amont de l'entrée dans la retenue et à une distance dite "de bon mélange ". II y a alors création d'un panache d'eau marquée qui individualise l'apport dont on pourra suivre la pénétration et la dispersion dans le lac.

La méthode consiste à injecter, à débit constant, plusieurs centaines de litres de la solution aqueuse du traceur, cette opération se faisant à l'aide d'une cuve à niveau constant ou d'une pompe doseuse (fig. 3).

L'ordre de grandeur des quantités de traceurs utilisés est de 10 à $30 \mathrm{li}$ tres pour les rhodamines et de 20 à 50 kilogrammes pour la fluorescéine et l'Amino $\mathrm{G}$ acid.

\subsubsection{L'injection instantanée}

Cette injection très brève permet la création d'un petit volume d'eau marqué en un point bien localisé afin de suivre son évolution dans le temps et l'espace (fig. 4). C'est le seul mode d'injection qui permette de déterminer les coefficients de dispersion.

L'utilisation d'un dispositif de dilution de la solution mère réduit les risques de création d'un gradient de densité. 


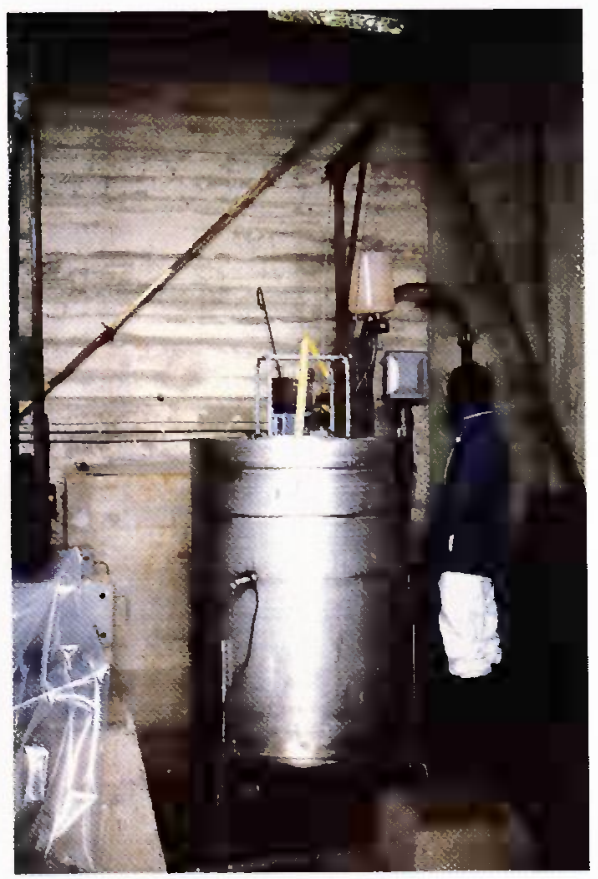

Fig. 3. - Injection continue de rhodamine dans la galerie de Bage.

Fig. 3. - Continuous thodamine injection in the Bage water gallery.

\section{II.3.3 Méthodologie de mesure}

Le dispositif de mesure comprend (fig. 2) :

- un ensemble de détection continue embarqué, qui permet la recherche et la détermination des dimensions des panaches d'eau marquée. Cet ensemble est constitué d'une ligne de mesure composée d'un tuyau gradué lesté à son extrémité, d'une électropompe de circulation de l'eau dans la ligne et d'un ou deux fluorimètres équipés des filtres optiques adaptés aux traceurs fluorescents utilisés (fig. 3). Par ailleurs, des échantillons d'eau sont systématiquement prélevés au rejet de cette ligne afin de procéder à des mesures précises des concentrations en traceurs au laboratoire ;

- un réseau de préleveurs automatiques d'échantillons d'eau flottants, modulables en position et en profondeur, dans le but de relayer l'embarcation durant la nuit et de faire des mesures précises en des points fixes bien définis (fig. 5);

- un dispositif de laboratoire constitué de fluorimètres équipés pour des mesures statiques des concentrations en traceurs dans les échantillons d'eau prélevés manuellement ou automatiquement ;

- un dispositif de localisation constitué d'un appareil optique (géodimètre AGA 140) émettant un rayon infrarouge qui se réfléchit sur une couronne de prismes installée sur l'embarcation (fig. 5). Après réflexion du rayon, le théodolite permet de mesurer les angles et la distance oblique du bateau. L'emplacement du géodimètre est lui-même repéré au préalable par une visée issue d'un point géodésique. Ce dispositif permet, par liaison radio, de positionner l'embarcation d'exploration et de suivre la progression du panache (Pujo, 1994).

Lorsque deux campagnes de traçages se sont succédées à des intervalles de temps suffisamment courts pour qu'un traceur résiduel puisse subsister dans la masse d'eau, une cartographie de traceur «point zéro» a été réalisée au préalable. 

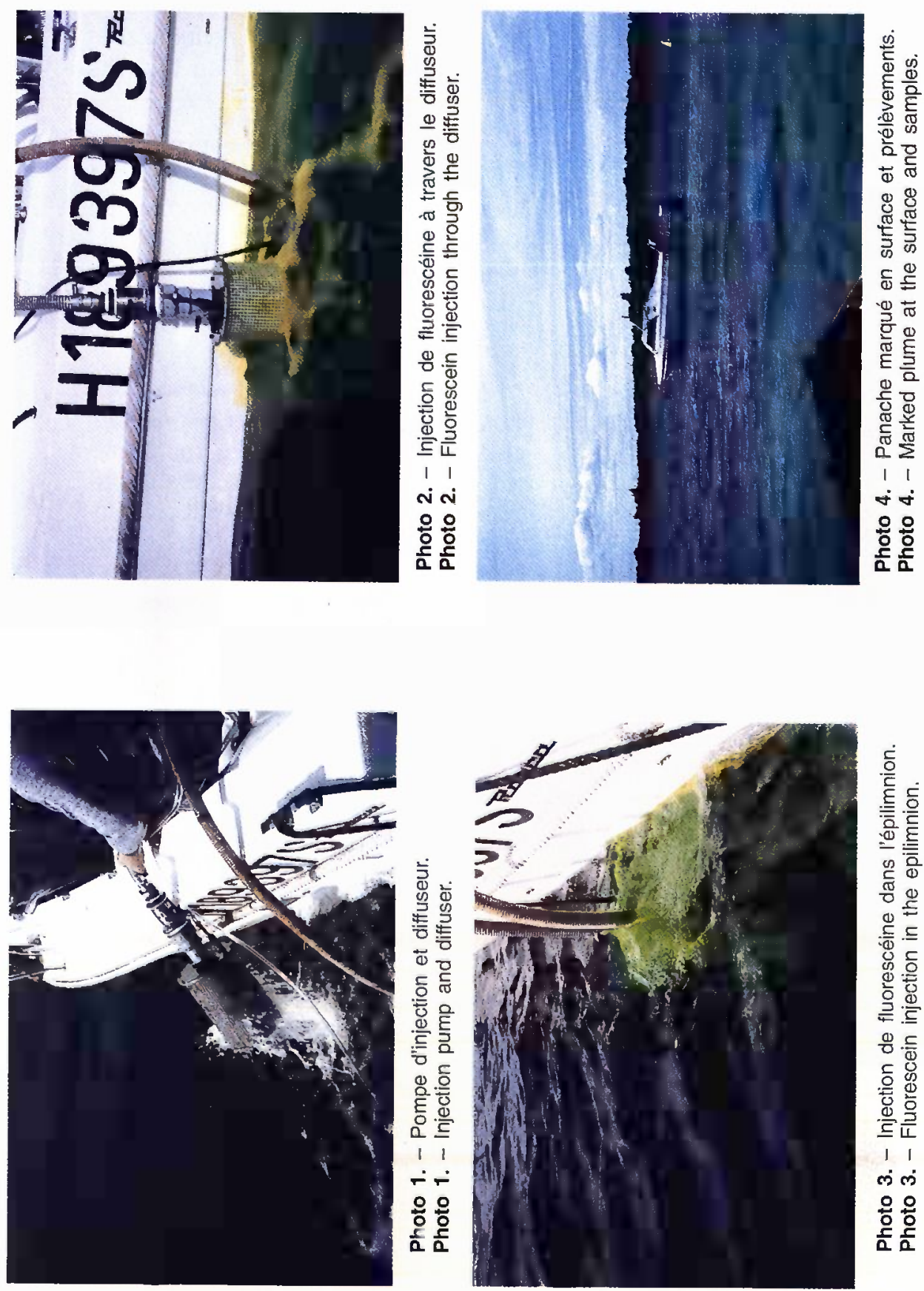

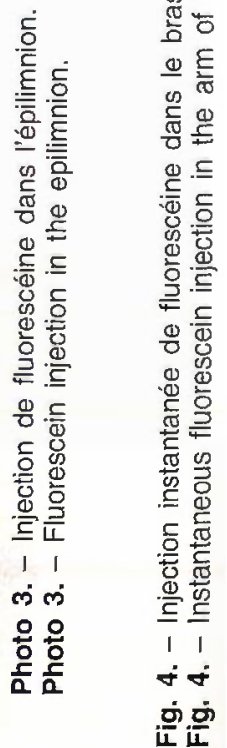



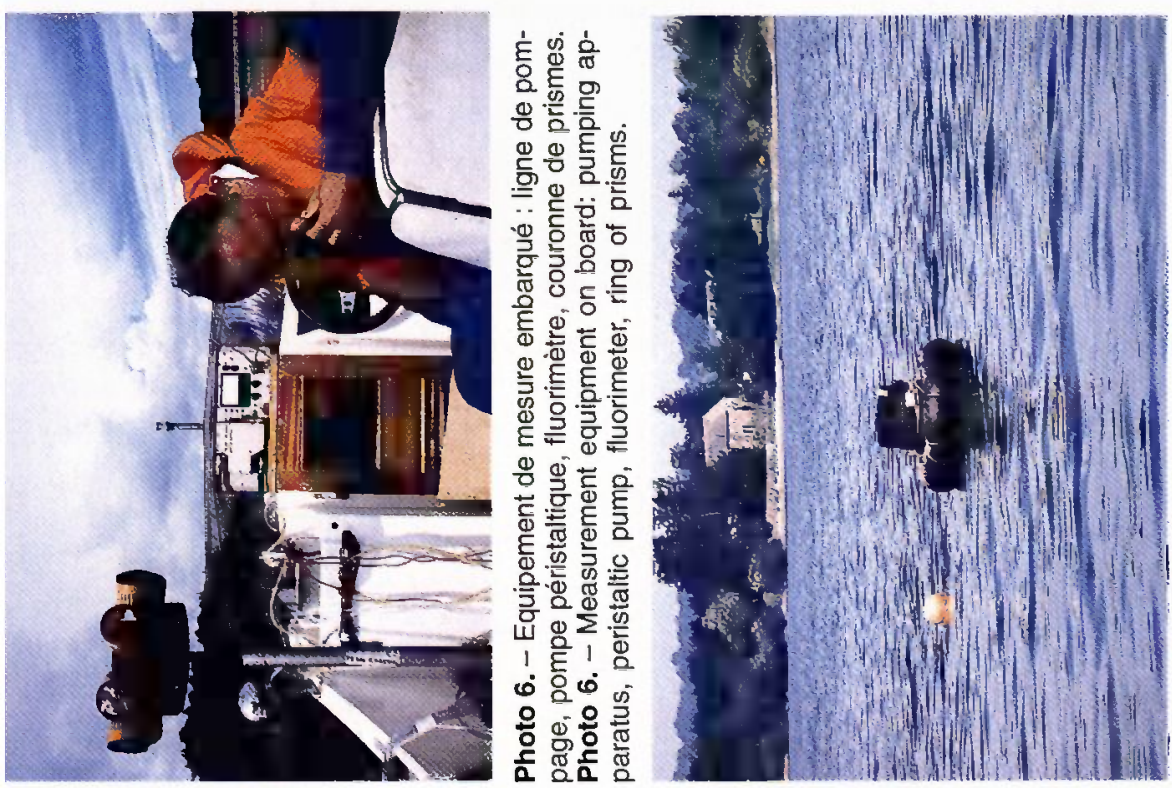

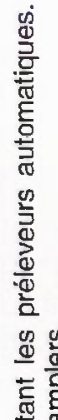

동

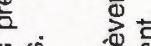

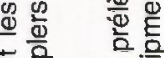

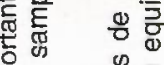

윽을

के है क

䒕苛婴
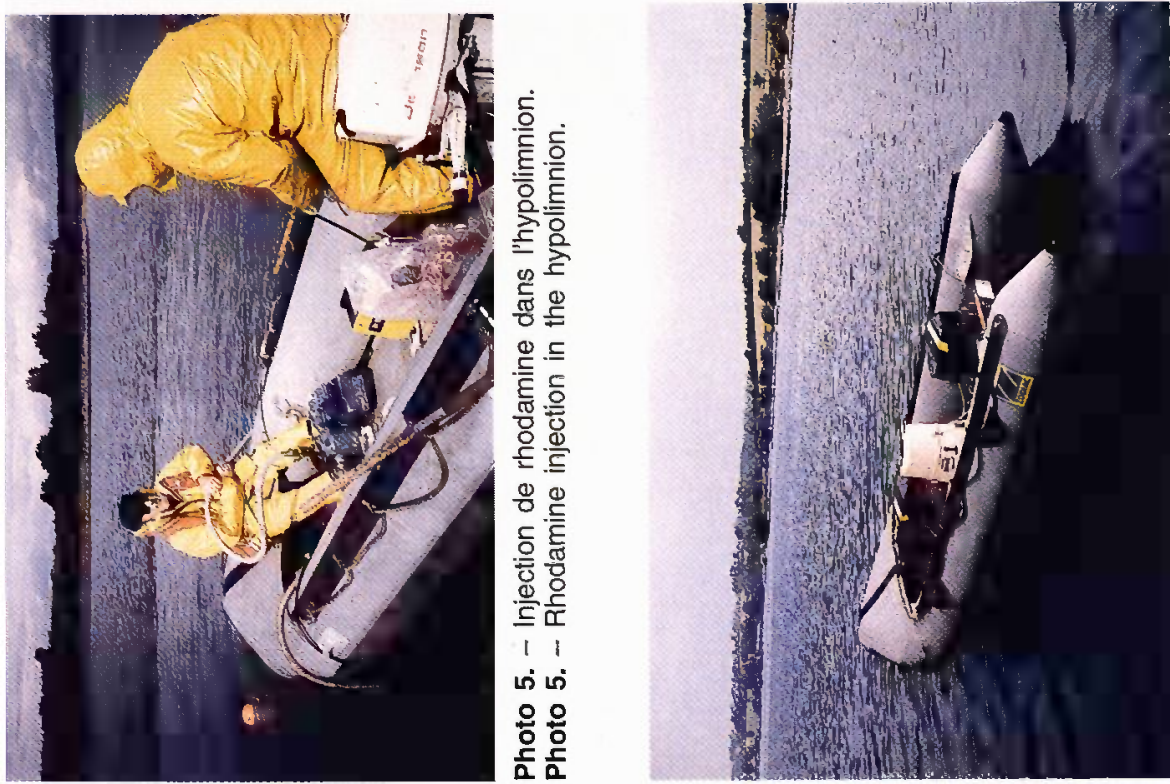

I I

เก เั

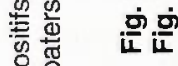

으는

I

$1 \infty$

$\infty$

แ

N

옹ㅇㅇ

을옹

돔 


\section{RÉPONSE D'UNE MASSE D'EAU À UN COUP DE VENT}

$\mathrm{Au}$ début d'un coup de vent, une masse d'eau, brusquement soumise à un champ de pression de surface, se déforme pour résister à cette contrainte. Une telle perturbation se traduit, au sein de la masse d'eau, par la propagation d'une série d'ondes qui mettent le fluide en mouvement, sollicitant le frottement et les forces d'inertie pour équilibrer les contraintes exercées par le vent. Les circulations ainsi créées peuvent être classées en deux grandes catégories, qui dépendent de la structure interne de la densité (essentiellement liée à la température en eau douce) :

- lorsque le lac n'est pas stratifié, la température est uniforme et la densité du fluide ne dépend que de la pression hydrostatique: les surfaces isobares (d'égale pression) et isopycnes (d'égale densité) sont parallèles. La circulation est pilotée uniquement par la déformation de la surface libre et elle intéresse la totalité de la masse d'eau. Cet écoulement est appelé barotrope;

- lorsque le lac est stratifié, la densité du fluide dépend de la pression hydrostatique mais aussi de la température. Les surfaces de discontinuité interne de densité (la thermocline, par exemple) se déforment pour compenser les gradients de pression, comme le fait la surface libre, ce qui crée un gradient horizontal de densité : les surfaces isobares et isopycnes ne sont plus parallèles. Ce sont alors les forces d'Archimède qui dominent l'écoulement, qui est appelé barocline.

Nous allons décrire brièvement les situations rencontrées, qui dépendent de la stratification thermique de la masse d'eau ainsi que de la force et de la durée du coup de vent.

Dans un milieu fermé comme un lac, ces circulations sont expliquées par l'effet de paroi. L'eau de surface mise en mouvement par le coup de vent s'accumule contre la berge du lac située sous le vent, élevant la cote du plan d'eau (fig. 6.1A et 6.2A). Cependant, en raison de la conservation de la masse, ce flux ne peut s'arrêter contre le bord. Aussi, l'eau plonge le long du bord sous le vent alors qu'une " source " apparaît le long de la berge située au vent (fig. 6.1B).

Cette résurgence correspond à une circulation sur le fond de la cuvette lorsque le lac est homogène, créant un écoulement barotrope sous la forme d'une grande boucle (fig. 6.1C).

Lorsque le lac est stratifié, la thermocline joue le rôle de "frontière mobile". Au début du coup de vent, sous l'effet de l'augmentation de pression créée par l'accumulation de l'eau de surface contre le bord, la thermocline bascule: elle s'enfonce sous le vent et se rapproche de la surface contre la berge au vent (fig. 6.2B). Ce basculement de la thermocline représente la façon dont le lac tente de compenser le champ de pression du vent en surface en créant, par la déformation des isothermes, un champ horizontal de pression interne au niveau de la thermocline : c'est une réponse baro- 

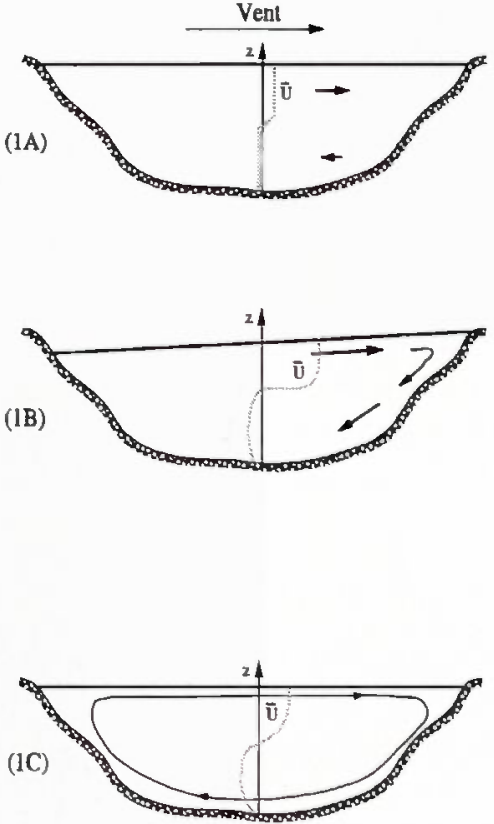

LAC HOMOGENE
Fig. 6.1 - Formation de mouvements barotropes dans un lac homogène soumis à un coup de vent.

(A) démarrage du mouvement, (B) mise en mouvement de toute la masse d'eau, (C) circulation barotrope stationnaire par boucle verticale.

Fig. 6.1 - Formation of barotropic motions in a homogeneous lake exposed to wind stress.

(A) initiation of motion, (B) whole basin circulation, (C) steady state barotropic circulation with one closed gyre.

Fig. 6.2 - Formation de mouvements baroclines dans un lac stratifié soumis à un coup de vent (d'après Imberger, 1979 ; 1987).

(A) démarrage du mouvement, $(B)$ basculement de la thermocline pour créer un gradient de pression barocline, (C) circulation barocline stationnaire par boucles verticales, (D) upwelling.

Fig. 6.2 - Formation of baroclinic motions in a stratified lake exposed to wind stress.

(A) initiation of motion, (B) tilting of the thermocline to set up a hydrostatic pressure gradient, (C) steady state baroclinic circulation with two closed gyres, (D) upwelling.
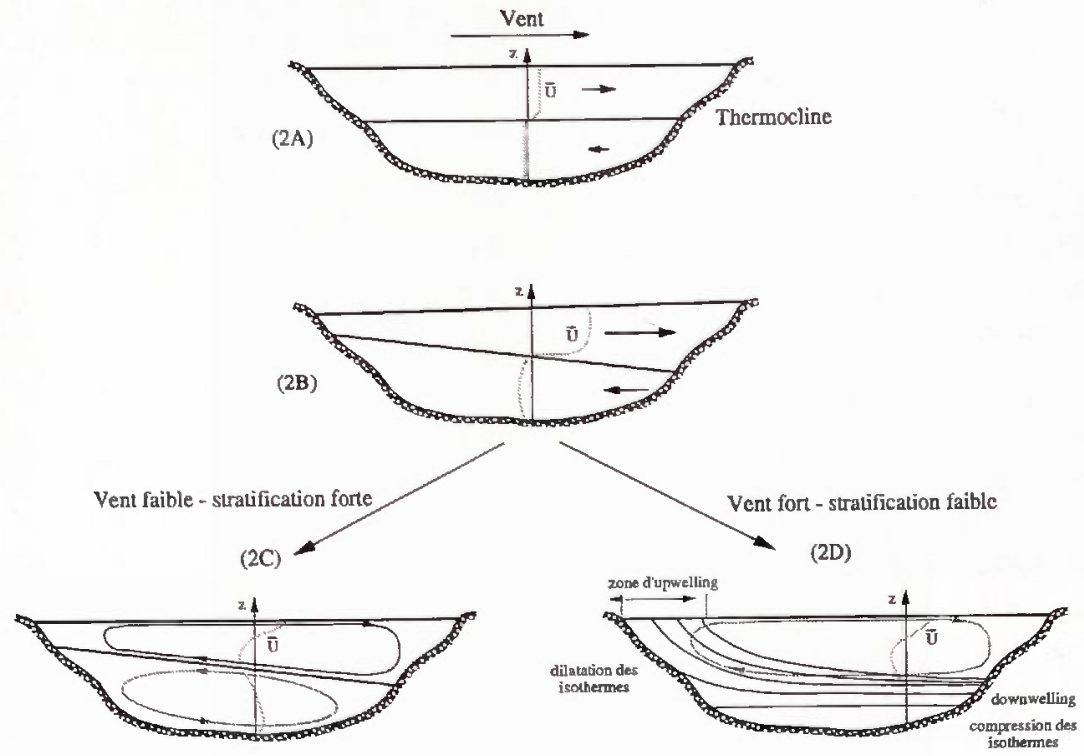

LAC STRATIFIE 
cline. L'eau résurgente provient alors du bas de l'épilimnion, créant un cisaillement de courant $\Delta U$ le long de cette interface. Par continuité, l'eau de I'hypolimnion proche de la thermocline est entraînée par le courant. Une circulation s'établit dans l'hypolimnion, symétrique de celle de l'épilimnion par rapport à la thermocline (fig. 6.2C).

Le temps caractéristique d'installation de cette circulation est donné par la période de seiche de mode 2 , $T_{i} \cdot \frac{T_{i}}{2}$ représente le temps que met une perturbation pour se propager d'un bord à l'autre, le long de l'interface, à la vitesse, $C_{i}$. Si $L$ est la longueur du lac dans le sens du vent, $T_{i}=\frac{2 L}{C_{i}}$ soit $T_{i}=\frac{2 L}{\sqrt{g \frac{\Delta \rho}{\rho} \frac{h(H-h)}{H}}}$. L'effet de bord ne sera pas ressenti au centre du bassin avant que l'onde de pression se déplaçant à la vitesse $C_{i}$ ne l'atteigne, soit tant que $t<\frac{T_{i}}{4}$.

Lorsque la rotation de la terre a un effet négligeable, cette circulation par boucles verticales a lieu dans un plan vertical dont l'axe horizontal est celui du sens du vent. Une telle circulation, lorsqu'elle parvient à s'établir, contribue à maintenir la structure verticale unidimensionnelle du lac, même si le cisaillement érode la thermocline. Par contre, si la force du vent ne peut être compensée par les forces de pression, la thermocline continue à basculer jusqu'à atteindre la surface contre la berge au vent, ce qui aboutit à une remontée en surface des eaux profondes. Ce phénomène est appelé upwelling. Imberger et Monismith (1986) ont montré que cet upwelling est accompagné d'un mouvement divergent qui étale les isothermes de la thermocline dans la zone de l'upwelling et les comprime dans la région opposée sous le vent (fig. 6.2D). Ainsi, dans la zone de l'upwelling, le gradient thermique est plus faible et la colonne d'eau exposée au vent, moins stable. La turbulence due au vent mélange l'eau profonde à l'eau de surface et le lac est mélangé par cet upwelling dont le front progresse horizontalement dans le sens du vent.

C'est le nombre de Wedderburn (W) qui permet de déterminer la réponse de la couche de surface et de s'assurer qu'une perturbation locale en surface se traduise par une réponse unidimensionnelle (verticale) du lac, sans entraîner de variations horizontales (Patterson et al., 1984). Il est basé sur la schématisation en deux couches de densité $\rho$ et $\rho+\Delta \rho$ où $g^{\prime}$ est la gravité réduite $\left(g^{\prime}=g \frac{\Delta \rho}{\rho}\right), h$ la hauteur de la couche de mélange, u* la vitesse de frottement de l'eau en surface, $L$ la longueur du lac dans le sens du vent.

$W$ exprime le rapport entre la force appliquée par le vent en surface $\left(U_{*}^{2} L\right)$ et les forces de pression barocline maximales au point d'upwelling $\left(g^{\prime} h^{2}\right)$, ces deux forces étant calculées par unité de surface du lac.

$$
W=\frac{\Delta \rho \mathrm{gh}^{2}}{\rho \mathrm{u}^{2} \mathrm{~L}}
$$

Pour les fortes valeurs de $W$ $(W>10)$, les variations horizontales 
de la hauteur de la couche de mélange, induites par le vent, sont faibles, donc il en est de même pour la production de turbulence par cisaillement à la thermocline. Dans cette situation, qui correspond à une stratification marquée et à des vents faibles, l'enfoncement de la couche de mélange est dominé par la turbulence produite en surface et transportée à la base de la couche de mélange où elle érode la thermocline. Les processus unidimensionnels dominent.

Pour des valeurs intermédiaires de $W(3<W<10)$, qui correspondent à une stratification marquée et à des vents forts, la thermocline bascule sous l'effet du vent, la production de cisaillement devient de plus en plus importante mais la structure unidimensionnelle est conservée. C'est dans ce cas que se produisent les circulations par boucles verticales.
Pour les faibles valeurs de $W$ $(W<3)$, les vents sont forts et la thermocline proche de la surface. Upwelling et mélange horizontal deviennent importants. L'approfondissement de la thermocline est dominé par le cisaillement qui agit à une échelle de temps plus faible que l'advection horizontale dans la couche de surface qui tente en vain de rétablir l'homogénéité horizontale. La thermocline, fortement inclinée, atteint alors la surface, ce qui crée un upwelling important.

Nous allons calculer, à trois périodes caractéristiques (printemps, été et automne), le nombre de Wedderburn ainsi que la période de seiche correspondante avec les caractéristiques du lac de Pareloup:

- largeur $B=500 \mathrm{~m}$;

- longueur totale $L=6000 \mathrm{~m}$;

\begin{tabular}{|c|c|c|c|c|c|c|c|c|c|}
\hline \multirow[b]{2}{*}{$\begin{array}{l}U \text { vitesse du vent } \\
\text { en } \mathrm{m}^{-1} \mathrm{~s}^{-1}\end{array}$} & \multicolumn{3}{|c|}{ Printemps } & \multicolumn{3}{|c|}{ Eté } & \multicolumn{3}{|c|}{ Automne } \\
\hline & 2 & 5 & 10 & 2 & 5 & 10 & 2 & 5 & 10 \\
\hline$h$ en $m$ & \multicolumn{3}{|c|}{9} & \multicolumn{3}{|c|}{10} & \multicolumn{3}{|c|}{12} \\
\hline Tépi en ${ }^{\circ} \mathrm{C}$ & \multicolumn{3}{|c|}{14} & \multicolumn{3}{|c|}{25} & \multicolumn{3}{|c|}{17} \\
\hline Thypo en ${ }^{\circ} \mathrm{C}$ & \multicolumn{3}{|c|}{11} & \multicolumn{3}{|c|}{11} & \multicolumn{3}{|c|}{12} \\
\hline$g^{\prime}$ & \multicolumn{3}{|c|}{$2,94 \cdot 10^{-3}$} & \multicolumn{3}{|c|}{$2,28 \cdot 10^{-2}$} & \multicolumn{3}{|c|}{$6,87 \cdot 10^{-3}$} \\
\hline$T_{i}$ en $h(L=6000 \mathrm{~m})$ & \multicolumn{3}{|c|}{23,3} & \multicolumn{3}{|c|}{8,1} & \multicolumn{3}{|c|}{13,9} \\
\hline Ti en $h(L=3000 \mathrm{~m})$ & \multicolumn{3}{|c|}{11,6} & \multicolumn{3}{|c|}{4} & \multicolumn{3}{|c|}{7} \\
\hline $\begin{array}{l}\text { Wedderburn W } \\
(L=6000 \mathrm{~m})\end{array}$ & 5,9 & 0,9 & 0,25 & 56,6 & 9 & 2,3 & 24,5 & 3,9 & 1 \\
\hline $\begin{array}{l}\text { Wedderburn } W \\
(L=3000 \mathrm{~m})\end{array}$ & 11,8 & 1,8 & 0,5 & 113,2 & 18 & 4,6 & 51 & 7,8 & 2 \\
\hline
\end{tabular}


- longueur jusqu'au détroit de Charouzech $\mathrm{L}=3000 \mathrm{~m}$;

- centre de volume du lac $L(H)=$ $9,75 \mathrm{~m}$;

- hauteur maximale $\mathrm{H}=40 \mathrm{~m}$.

Les résultats obtenus nous montrent que le nombre de Wedderburn est inférieur à 3 lorsque le vent est très fort. On peut donc s'attendre à être en présence d'upwellings par vent modérés au printemps, alors que la stratification n'est pas bien marquée. Cependant, la période de seiche est plus longue, à ce momentlà, ce qui implique une durée du coup de vent suffisamment longue pour que l'upwelling s'établisse. En été, le risque de développement des upwellings est pratiquement inexistant car les étés sont très peu ventés. L'automne présente des conditions favorables pour l'établissement de circulations par boucles verticales, productrices de turbulence par cisaillement, car le vent forcit en amplitude et en durée alors que le lac est bien stratifié.

\section{L'ÉTUDE DE LA RETENUE DE PARELOUP}

La retenue de Pareloup, est située sur le plateau du Lévezou, à une altitude de $800 \mathrm{~m}$. Sa superficie importante (1260 ha) est soumise à des vents parfois très violents qui induisent des circulations de masse d'eau importantes. La gestion hydraulique de stockage saisonnier comporte une entrée d'eau forcée en profondeur provenant d'un pompage dans la retenue de Bage et un prélèvement d'eau par turbinage situé lui aussi près du fond de la retenue. De plus, la forme du lac est assez découpée: le corps principal du lac, correspondant à la vallée inondée du Vioulou, est orienté Est-Ouest; perpendiculairement à cet axe, les anciens lits de petits ruisseaux (Rieutord, Connes...) forment des bras latéraux dont les trois principaux sont situés au Sud et orientés Nord-Sud (fig. 7). Une telle complexité topographique rajoute à la difficulté de connaître les échanges de masses d'eau dans cette retenue.

Nous avons procédé au traçage des masses d'eau de ce lac à l'aide des traceurs fluorescents, afin de mettre en évidence :

- la localisation et l'évolution du panache d'eau provenant de la retenue de Bage qui pénètre dans le fond de la retenue de Pareloup;

- les échanges d'eau entre un bras peu profond et le corps principal de la retenue;

- le régime de circulation des eaux de part et d'autre du détroit qui sépare la retenue en deux grandes parties Est et Ouest;

- la proportion de l'apport de Bage soutiré lors d'un turbinage immédiatement successif.

Ces campagnes de traçages ont eu lieu aux périodes de l'année déterminantes pour la stratification thermique: l'installation de la thermocline (juin) et la déstratification (septembre). 


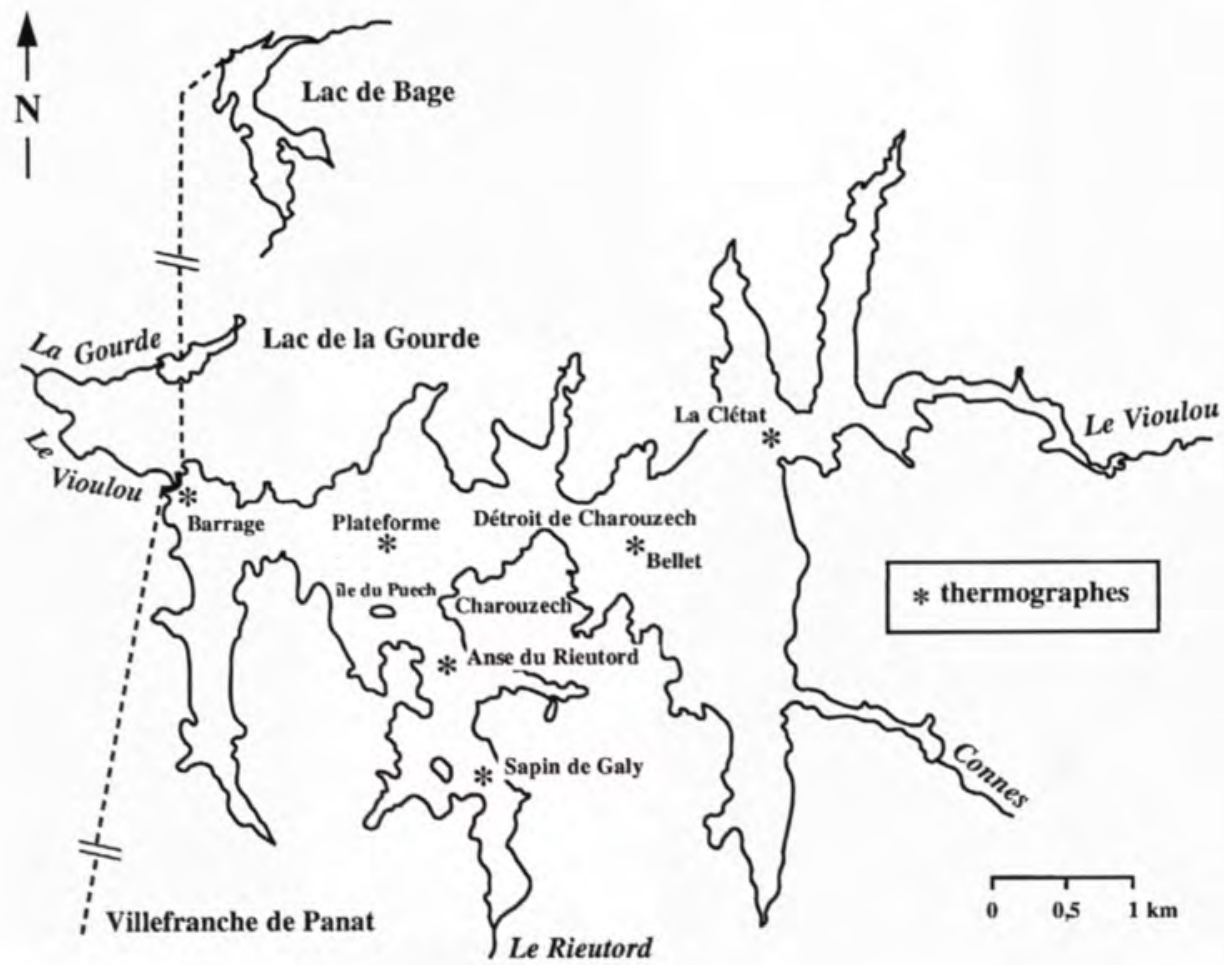

Fig. 7. - Plan de la retenue de Pareloup.

Fig. 7. - Map of Pareloup Lake.

\section{IV.1 Suivi du panache de l'eau de Bage}

La remontée par pompage des eaux de Bage a lieu pendant les périodes de faible demande énergétique, soit essentiellement la nuit et en fin de semaine. Nous avons suivi le panache lors de situations de fin de semaine, l'une en septembre 1987 (Salençon et al., 1988), l'autre en juin 1988 (Calmels et al., 1989a).

Les deux campagnes réalisées pour des débits de pompage de $6,3 \mathrm{~m}^{3} \cdot \mathrm{s}^{-1}$ en septembre (1 pompe) et $12 \mathrm{~m}^{3} \cdot \mathrm{s}^{-1}$ en juin ( 2 pompes) ont nécessité respectivement 20 et 54 litres de rhodamine $B$ dilués dans 200 et 450 litres d'eau qui ont été injectés en continu dans la conduite d'amenée durant 26 et 47 heures.

Le marquage a eu lieu à partir de la cheminée d'expansion de la canalisation d'amenée située à proximité du barrage à 100 mètres de l'exutoire. Cette conduite débouche ensuite dans la retenue à 37 mètres de profondeur à la cote 768 NGF.

Le suivi du panache d'eau marquée a commencé dès le début de 
son arrivée dans la retenue, et s'est poursuivi durant deux jours, le suivi par bateau étant interrompu pendant la nuit.

\section{IV.1.1 Traçage de septembre 1987}

Début septembre, le lac présente les conditions de stratification les plus stables: la thermocline est située à $-10 \mathrm{~m}$, la température de l'épilimnion est de $20^{\circ} \mathrm{C}$ alors que celle de l'hypolimnion est voisine de $12^{\circ} \mathrm{C}$. Les profils d'oxygène dissous mesurés au barrage avant le début du pompage montrent une oxycline calquée sur la thermocline, l'hypolimnion étant pratiquement anoxique à partir de $-12 \mathrm{~m}$ (fig. 8). Cette situation s'explique par le fait qu'en raison des travaux effec- tués au barrage, les usines ont été pratiquement à l'arrêt de fin mars à septembre, ce qui a considérablement limité le renouvellement des eaux du fond tout en accentuant la stratification thermique.

L'injection a eu lieu du 9 au 10 septembre pendant 25 heures avec un débit des pompes de $6,3 \mathrm{~m}^{3} \cdot \mathrm{s}^{-1}$ établi depuis 48 heures. L'eau provenant de Bage avait une température de $15,7^{\circ} \mathrm{C}$ et une teneur en oxygène dissous de $2,7 \mathrm{mg} \cdot{ }^{-1}$. Pendant toute la durée du traçage, le vent est resté faible.

Avec l'arrivée d'eau plus chaude, le panache se mélange à son niveau d'arrivée en réchauffant le fond du lac de $1^{\circ} \mathrm{C}$, à partir de la cote $-28 \mathrm{~m}$, puis, plus léger que l'eau avoisinante,

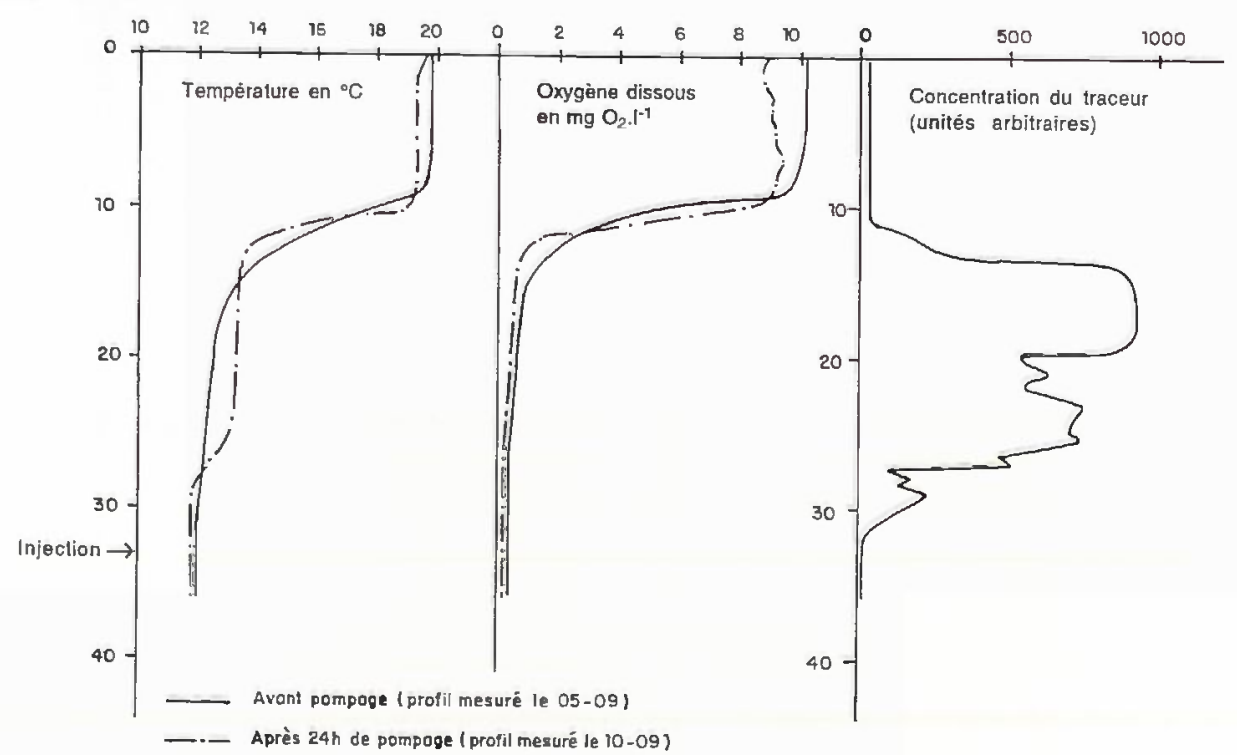

Fig. 8. - Profils de température, oxygène dissous et rhodamine mesurés au barrage les 5 et 10 septembre 1987.

Fig. 8. - Profiles of temperature, dissolved oxygen and rhodamine measured at the dam on September 5 and 10, 1987. 
tend à remonter (les volutes du panache sont bien matérialisées par le profil du signal fluorimétrique) (fig. 8). Cependant la remontée du panache est stoppée dès que sa densité correspond à la densité du lac : en raison du fort gradient thermique établi à cette période de l'année (environ $7^{\circ} \mathrm{C}$ sur $4 \mathrm{~m}$ à la thermocline) et de l'homogénéité de l'eau du fond, le panache remonte jusque sous la thermocline mais ne peut la franchir.

Au vu de sa forme, en plan et de profil, le terme de «langue» est le plus approprié pour représenter la forme du panache. Dans son allure générale, cette langue présente, dans tous les cas, un palier plus marqué sur sa face supérieure que sur sa face inférieure qui n'atteint jamais le fond de la retenue.

Le panache du rejet suit les thalwegs du Vioulou et de ses proches affluents en envahissant les zones les plus basses avec une vitesse variant de 170 à $100 \mathrm{~m} \cdot \mathrm{h}^{-1}$ selon la largeur du fond. La partie inférieure du panache ne rentre pas au contact du fond de la retenue et des thalwegs. La partie supérieure du panache prend la forme d'un palier quasi-horizontal qui remonte progressivement à une vitesse moyenne de $3 \mathrm{~m} \cdot \mathrm{h}^{-1}$, puis reste confiné très distinctement sous la thermocline située entre -10 et $-11 \mathrm{~m}$ les 9 et 10 septembre 1987 (fig. 9).

On constate que l'évolution du panache est guidée par les écarts de densité entre cette langue d'eau légèrement plus chaude injectée dans le lac et l'eau du lac avoisinante: plus léger, le panache a tendance à s'étendre horizontalement en remontant légèrement pour trouver son niveau de densité. La présence à cette période de l'année, d'une thermocline très marquée, permet de le localiser très distinctement, puisqu'il ne peut franchir cette barrière de densité. Mais, plus on s'éloigne du point d'injection, plus l'écart de densité devient faible, limitant l'ampleur du mélange vertical donc l'épaisseur du panache.

Pour un régime de rejet établi depuis 48 heures, à un débit de $6,3 \mathrm{~m}^{3} \cdot \mathrm{s}^{-1}$, le taux de dilution minimale observée au milieu du panache 25 heures après le début de l'injection du traceur est égal à 12 et le taux de dilution moyen du panache dans la retenue est de l'ordre de 20. Le taux moyen de dilution calculé à partir du rapport des volumes, recoupe celui calculé à partir des concentrations en traceur observées à la plateforme de mesures, ce qui confirme une épaisseur moyenne de 10 mètres pour le panache durant les deux jours d'expérimentation.

\section{IV.1.2 Traçage de juin 1988}

À cette période de l'année, la stratification de la retenue était faible $\left(\Delta \mathrm{T}=3^{\circ} \mathrm{C}\right.$ entre $-10 \mathrm{~m}$ et $\left.-11 \mathrm{~m}\right)$ et le débit de pompage était maximal $\left(12 \mathrm{~m}^{3} \cdot \mathrm{s}^{-1}\right)$. L'injection a eu lieu du 4 au 6 juin, durant 47 heures.

L'eau provenant de Bage était à une température de $12,7^{\circ} \mathrm{C}$ et a pénétré dans un hypolimnion à $11^{\circ} \mathrm{C}$. Cette eau plus légère est remontée par effet de densité et le panache est venu buter sous la thermocline, vers 


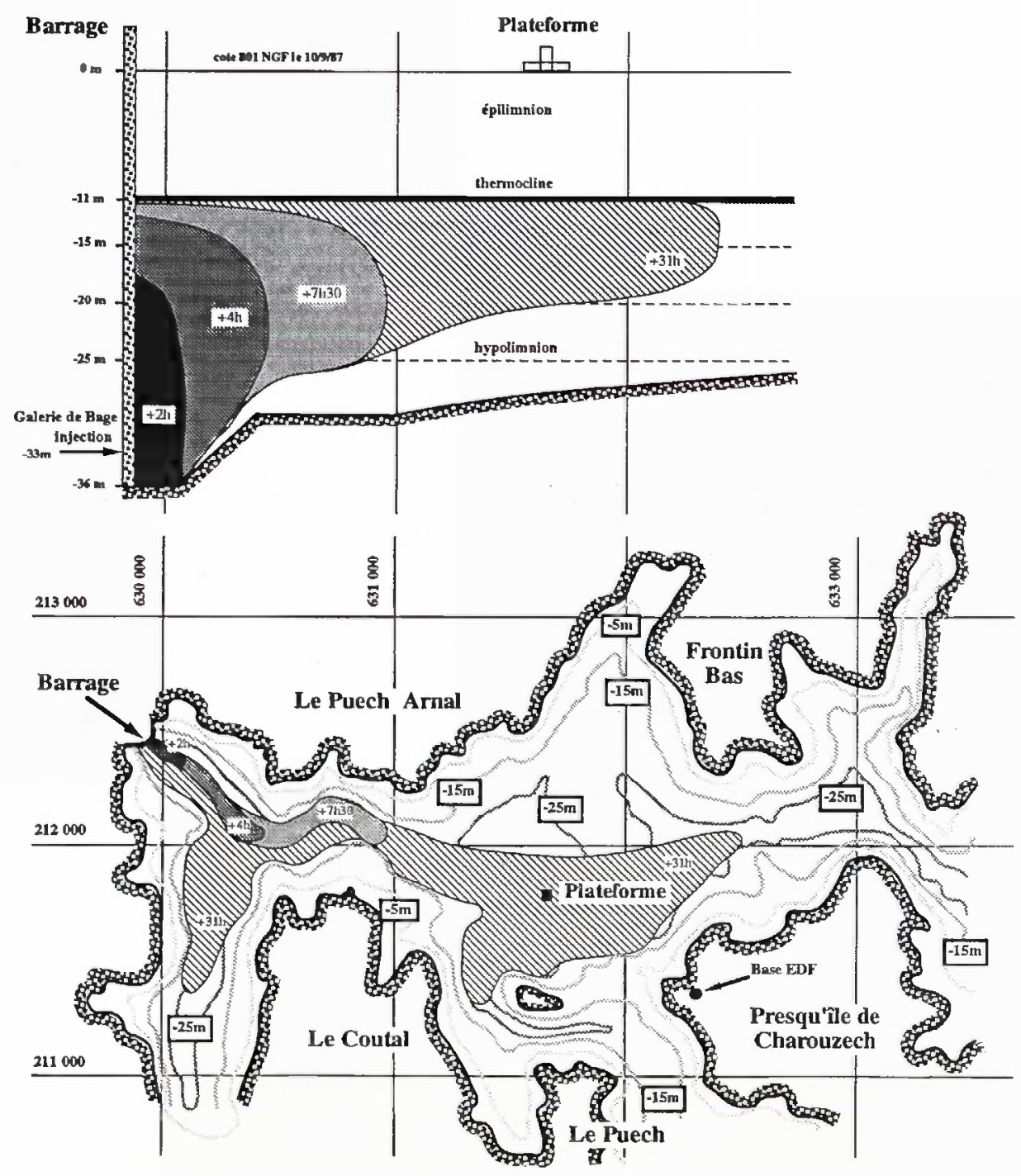

Fig. 9. - Situation du panache le 10 septembre 1987.

Fig. 9. - State of the plume on September 10, 1987.

$-10 \mathrm{~m}$. Le panache a ensuite pénétré dans les bras latéraux tout en restant sous la thermocline, avec une vitesse maximale comprise entre $180 \mathrm{~m} \cdot \mathrm{h}^{-1}$ à proximité de l'émissaire et $72 \mathrm{~m} \cdot \mathrm{h}^{-1}$ au-delà de la plateforme et dans les bras latéraux.

Le comportement du panache dans ces conditions de printemps (faible stratification thermique et débit de 

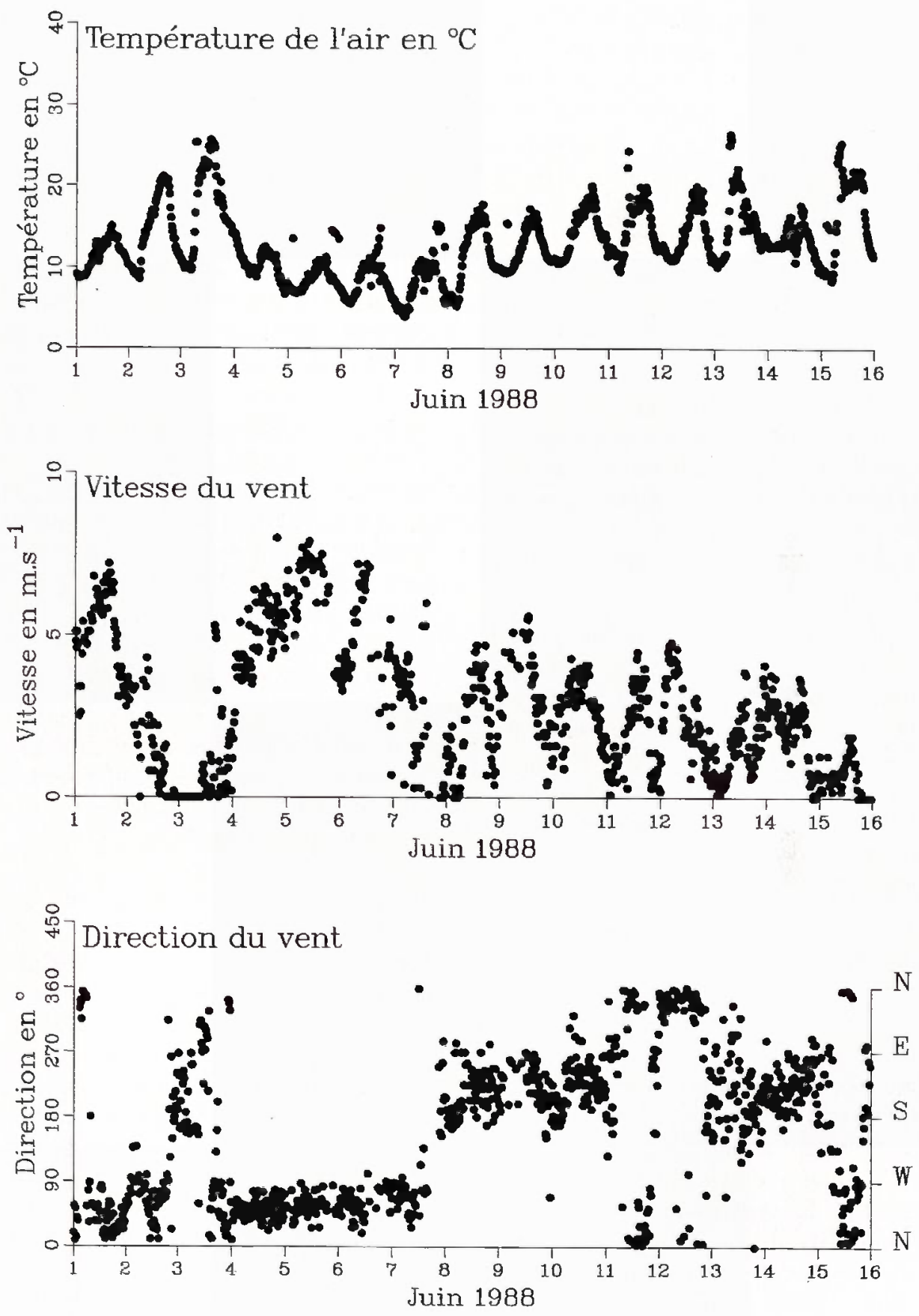

Fig. 10. - Température de l'air, vitesse et direction du vent mesurés sur la plateforme. Juin 1988.

Fig. 10. - Air temperature, speed and direction of wind measured from the platform. June 1988. 
pompage maximal de $12 \mathrm{~m}^{3} \cdot \mathrm{s}^{-1}$ pendant 48 heures) confirme celui observé lors de la campagne de septembre 1987 dans des conditions d'automne (très forte stratification thermique et débit de pompage réduit à $6 \mathrm{~m}^{3} \cdot \mathrm{s}^{-1}$ pendant 24 heures) : la thermocline joue le rôle de barrière de densité, l'eau injectée reste confinée dans l'hypolimnion et évolue horizontalement en suivant la bathymétrie jusque dans les bras latéraux du lac.

Cependant, lors de cette campagne, nous avons eu la chance de bénéficier de conditions météorologiques particulières qui nous ont permis de mettre en évidence des phénomènes extrêmement importants pour l'écosystème.

Au cours des jours qui ont précédé le traçage, les 2 et 3 juin, le vent était très faible. Les profils thermiques mesurés le 2 juin montrent un échauffement très important en surface. Le 4 juin juste avant le début de l'injection, un vent de Nord-Ouest a commencé à souffler avec une vitesse importante de $5 \mathrm{~m} \cdot \mathrm{s}^{-1}$ (fig. 10).

Les faibles valeurs à cette période du nombre de Wedderburn $(W=0,9)$ ainsi que du nombre de Lac $\left(L_{N}=0,24\right)$ suggèrent la possibilité d'une remontée des eaux de l'hypolimnion en surface (Salençon, 1994). L'examen des isothermes tracées à partir des mesures (fig. 11) confirme très nettement l'installation d'un upwelling, la remontée des eaux profondes ayant lieu contre le barrage. Cet upwelling s'accompagne d'une dilatation des isothermes contre le barrage : les profils thermiques relevés le
5 juin montrent bien que la thermocline est remontée de $-11 \mathrm{~m}$ à $-2 \mathrm{~m}$ près du barrage, le gradient thermique diminuant de $3^{\circ} \mathrm{C}$ à $1,5^{\circ} \mathrm{C}$. De plus, elle présente horizontalement une pente régulière du barrage vers la plateforme (fig. 12).

Les profils de rhodamine mesurés régulièrement à la bouée "barrage" montrent la remontée du panache de rhodamine en surface à partir du 5 juin. Le temps d'installation de l'upwelling correspond à celui de la période de seiche calculée avec la structure thermique, soit $24 \mathrm{~h}$. Ces eaux marquées sont alors entraînées dans le courant de surface par le vent vers la plateforme où leur passage est signalé par une augmentation de la concentration de rhodamine à $-0,5 \mathrm{~m}$ et $-5 \mathrm{~m}$ le 5 juin à partir de $19 \mathrm{~h}$.

Il est à noter que la présence de l'upwelling au barrage n'empêche pas la progression du panache vers la plateforme et au-delà.

Cette situation dure jusqu'à ce que le vent tourne au Sud-Est, le 7 juin au soir (fig. 10). Alors, on constate que l'upwelling s'inverse. L'eau chaude s'accumule en surface contre le barrage avec un approfondissement considérable de la thermocline vers $-15 \mathrm{~m}$ et un resserrement des isothermes (fig. 11). Le panache de rhodamine est alors repéré plus bas, vers $-20 \mathrm{~m}$, à la bouée $\mathrm{C}$, entre la plateforme et le barrage. Les préleveurs de la plateforme indiquent que le panache de rhodamine qui était à $-15 \mathrm{~m}$ est repéré à $-25 \mathrm{~m}$ à partir du 9 juin 

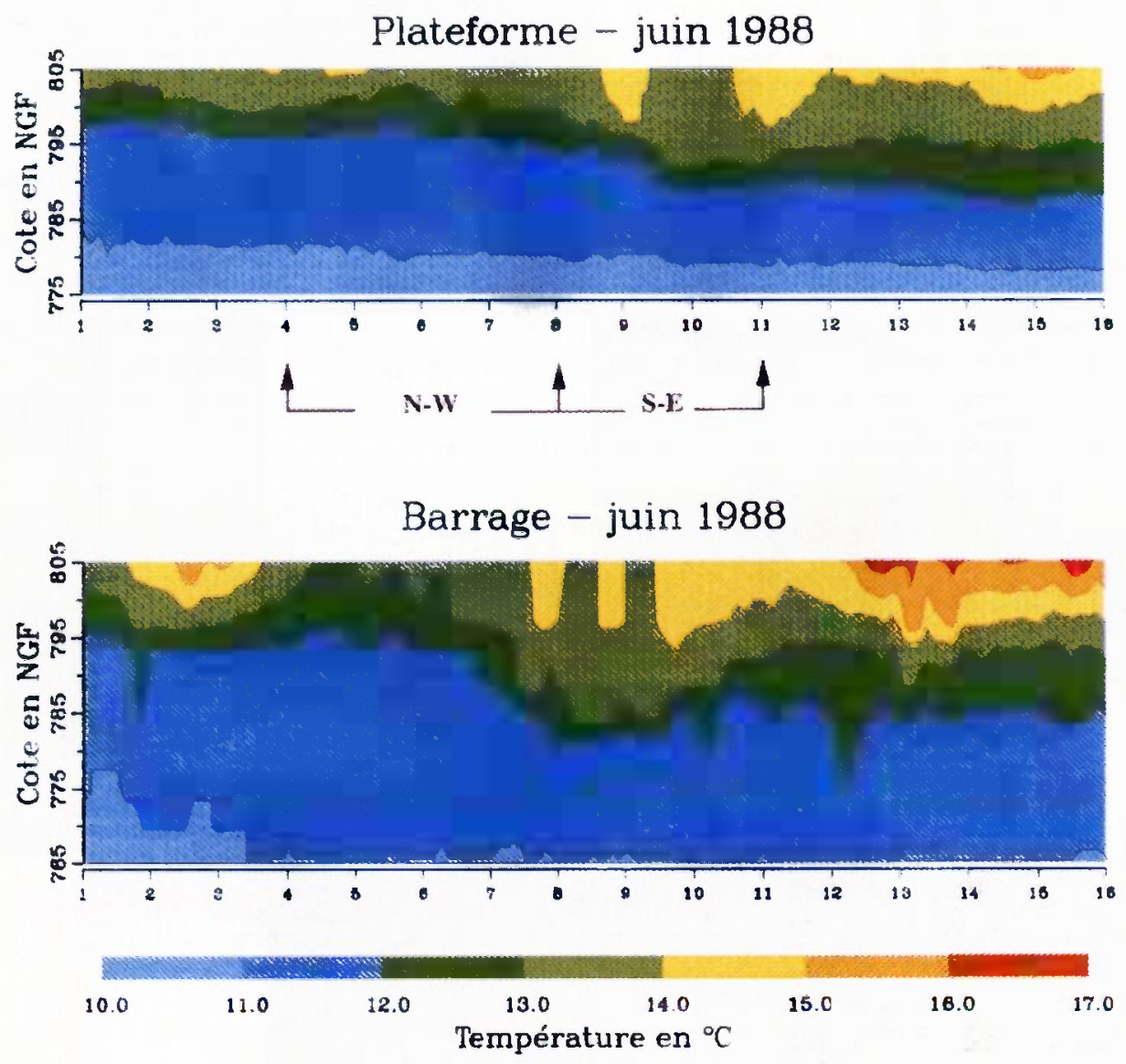

Fig. 11. - Isothermes mesurés au barrage et à la plateforme. Juin 1988.

Fig. 11. - Isotherms measured at the dam and the platform. June 1988.

à $19 \mathrm{~h}$ et il est remplacé par de l'eau de surface à $-15 \mathrm{~m}$. Cette situation apparaît $24 \mathrm{~h}$ environ après le changement de vent, ce qui correspond là aussi à la période de seiche.

L'eau marquée à la rhodamine réapparaît en surface dans le fond de la baie du Rieutord, où a lieu l'upwelling contre la berge par vent de Sud, que l'on repère bien sur les iso- thermes (fig. 13). L'augmentation des concentrations dans le fond de la baie est observée le 8 juin entre $10 \mathrm{~h}$ et $12 h$, soit 12 heures après que le vent a tourné. Alors, l'eau du fond de ce bras contient $3 \%$ de l'eau de Bage, soit un coefficient de dilution de 33 .

Cette campagne de traçage a permis de mettre en évidence que, sous des conditions de stratification faible 

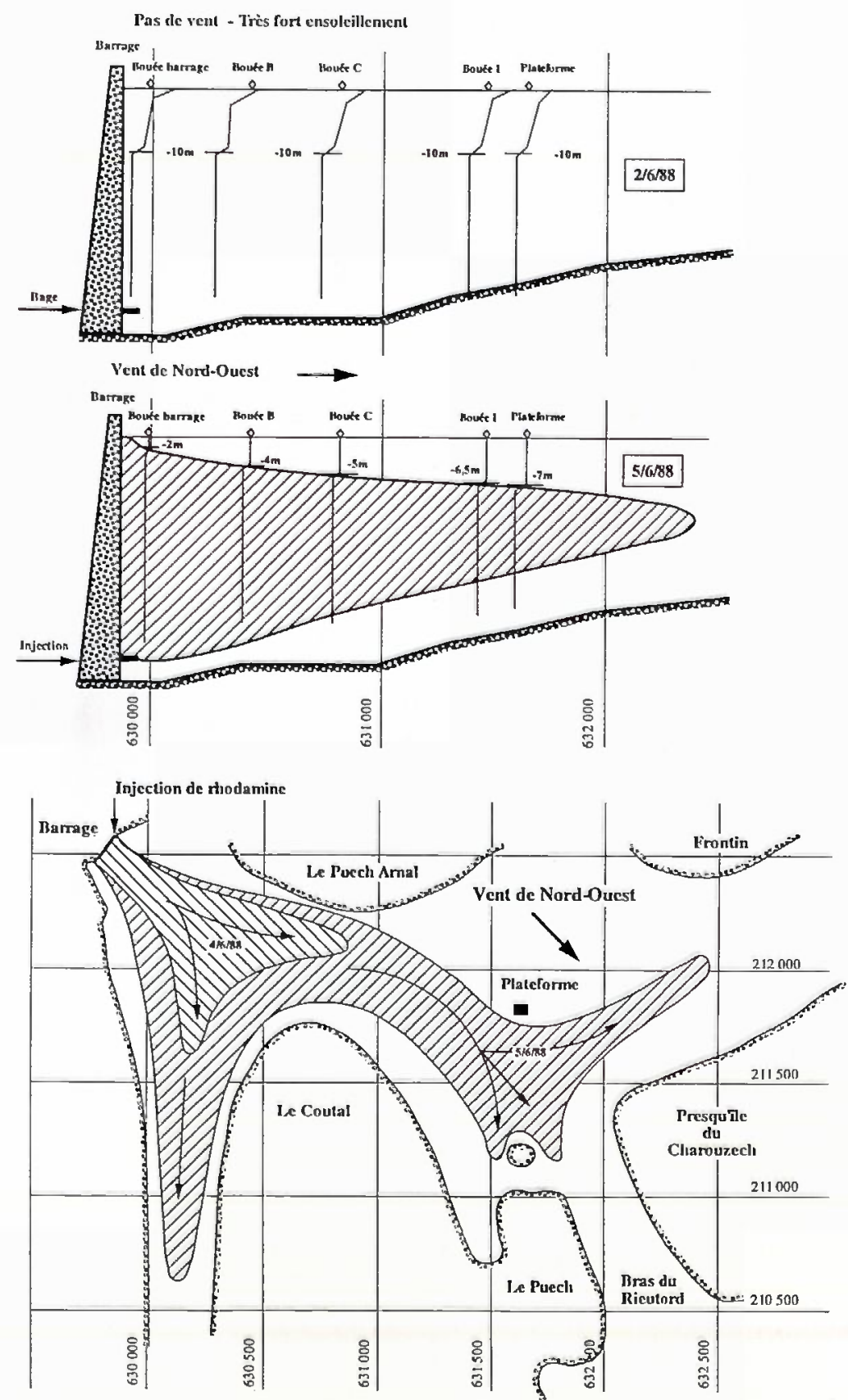

Fig. 12. - Marquage de l'upwelling par vent de Nord-Ouest.

Fig. 12. - Tracing of the upwelling with a north-westerly wind. 

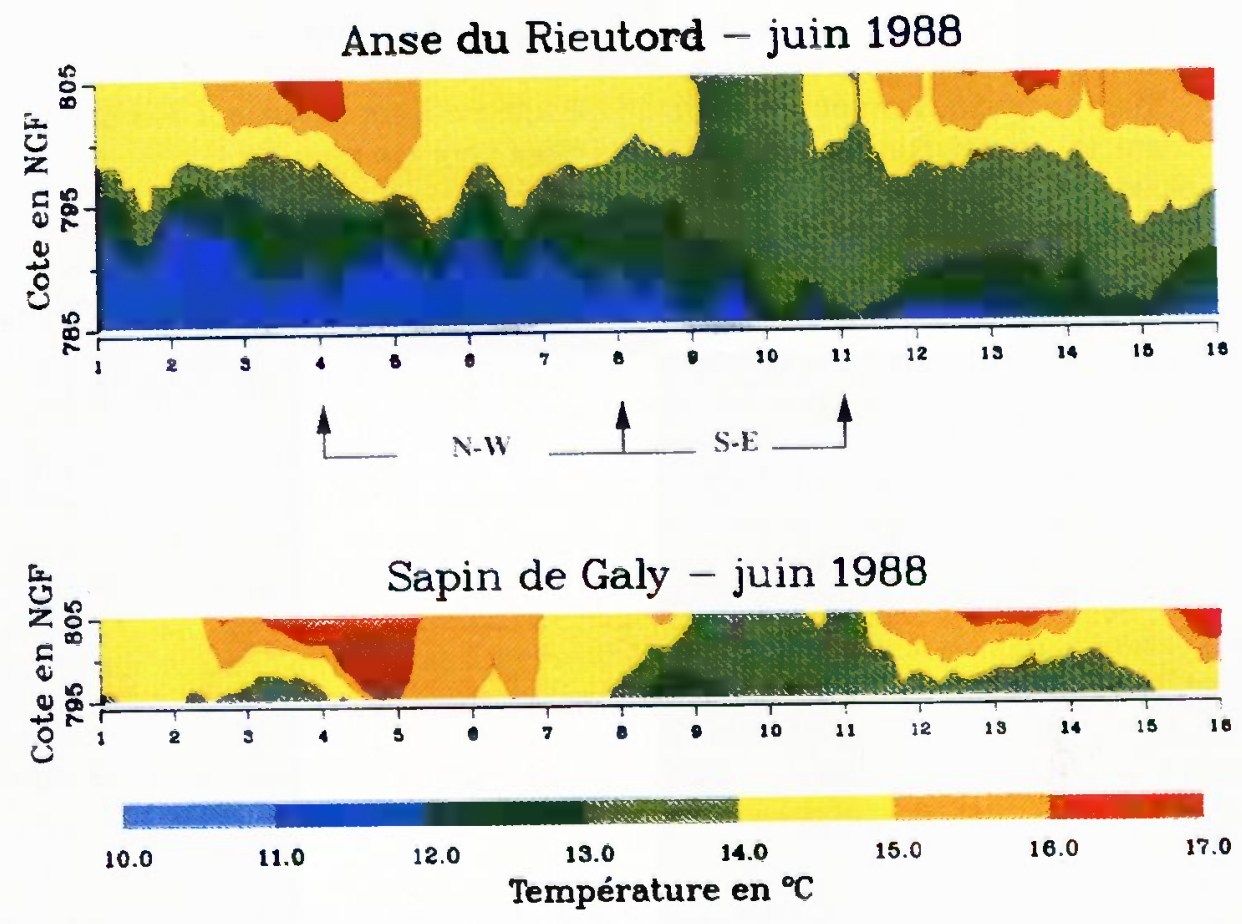

Fig. 13. - Isothermes mesurés dans le bras du Rieutord. Juin 1988.

Fig. 13. - Isotherms measured in the arm of the Rieutord. June 1988.

et lors des coups de vents successifs qui interviennent couramment au printemps, le lac de Pareloup est sujet à des upwellings qui apportent en surface une part non négligeable des apports de Bage.

II est certain que ces conditions, constituent une configuration de réalimentation des eaux de surface en nutriments, les apports de Bage étant les plus riches, à une période où le bloom de diatomées a épuisé le stock de nutriments des eaux superficielles. Le temps de mise en place de ces upwellings est de l'ordre de 12 à $24 \mathrm{~h}$ selon la stratification établie et la to- pographie du lac, ce qui permet à des coups de vent relativement courts d'amorcer ce phénomène de remontée d'eaux profondes.

\section{IV.1.3 Conclusion}

Le suivi du panache de l'eau pompée à Bage a eu lieu dans deux situations caractéristiques: au printemps, par faible stratification, avec un pompage de fin de semaine (2 pompes soit $12 \mathrm{~m}^{3} \cdot \mathrm{s}^{-1}$ du vendredi soir au lundi matin) et à l'automne, en période de forte stratification, avec une seule pompe $\left(6,3 \mathrm{~m}^{3} \cdot \mathrm{s}^{-1}\right)$. 
Dans les deux cas, le panache s'est étiré progressivement au-dessus des thalwegs jusqu'au détroit central, prenant la forme d'une langue. Son évolution a été guidée par la bathymétrie et les écarts de densité entre cette langue d'eau plus chaude injectée dans le lac et l'eau environnante. Plus léger, le panache a eu tendance à s'étendre horizontalement en remontant légèrement pour trouver son niveau de densité. La partie inférieure du panache n'est pas venue au contact du fond. Si le panache n'a jamais franchi la thermocline au sens où il ne l'a pas détruite par convection, nous avons mis en évidence une situation d'upwelling au printemps au cours de laquelle l'eau de l'hypolimnion est remontée en surface lors du

basculement de la thermocline par le vent. Ainsi, les eaux plus riches de Bage peuvent alimenter temporairement l'épilimnion.

Par ailleurs, dans les deux cas de rejet, la surface occupée 2 jours après le début du marquage était sensiblement la même, mais l'épaisseur du panache était plus importante pour un débit double.

Le taux de dilution moyen calculé à partir des nombreuses mesures de concentration était compris entre 19 pour un rejet de $6 \mathrm{~m}^{3} \cdot \mathrm{s}^{-1}$ et 12 pour un rejet de $12 \mathrm{~m}^{3} \cdot \mathrm{s}^{-1}$, au milieu de la partie Ouest de la retenue.

La durée importante (48 heures) du rejet était néanmoins insuffisante pour

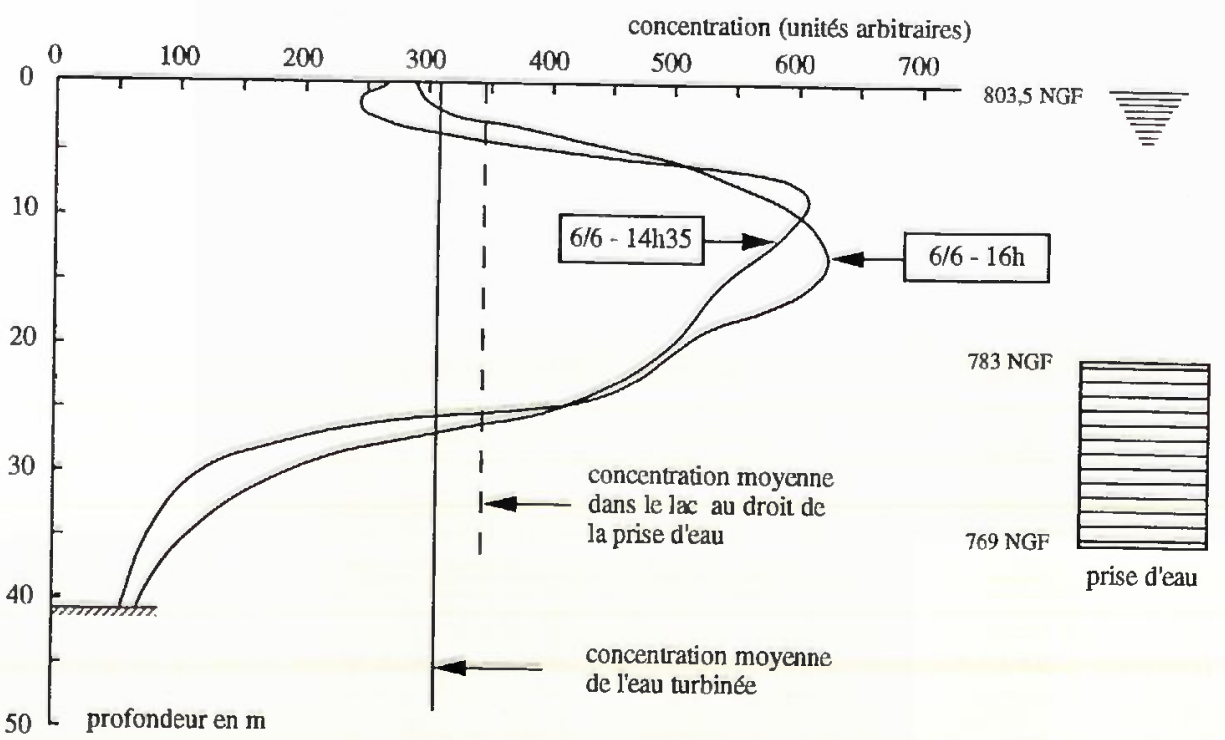

Fig. 14. - Concentrations en rhodamine dans le lac au droit de la prise d'eau et dans l'eau turbinée.

Fig. 14. - Rhodamine concentrations in the lake near the water inlet and in the turbined water. 
que son eau traverse le détroit et atteigne la partie Est de la retenue.

\section{IV.2 Turbinage successif à un pompage}

Le 6 juin 1988, après l'arrêt du pompage de Bage marqué à la rhodamine, l'eau a été reprise par turbinage pendant $12 \mathrm{~h}$ à un débit de $20 \mathrm{~m}^{3} \cdot \mathrm{s}^{-1}$.

Les concentrations en rhodamine mesurées dans la galerie d'Alrance indiquent que l'eau turbinée contient $6 \%$ d'eau de Bage, ce qui correspond à un facteur de dilution moyenne de l'eau pompée à Bage égal à 17. De plus, la concentration moyenne en rhodamine de l'eau soutirée est inférieure de $10 \%$ à la concentration moyenne mesurée sur la verticale au droit des vannes de fond au barrage (fig. 14).

Cette part relativement faible de l'eau de Bage est expliquée par la situation d'upwelling. Le panache étant remonté en surface contre le barrage, la prise d'eau prélève une eau peu marquée en rhodamine. II reste que, dans une situation "horizontale", le turbinage devrait prélever davantage d'eau de Bage.

\section{IV.3 Échange des masses d'eau entre la retenue et le bras du Rieutord}

Les fonds peu profonds des bras latéraux jouent un rôle prépondérant pour la reproduction des espèces piscicoles.

Le bras du Rieutord, dont la section de jonction avec le corps de la retenue est rétrécie par la présence des hauts fonds de l'île du Puech, a été marqué à plusieurs reprises, afin d'étudier la complexité de ses échanges avec le reste de la retenue.

Le premier marquage a été réalisée en juin 1988, par vent de Sud (Calmels et al., 1989a). Comme nous l'avons vu précédemment, un upwel-

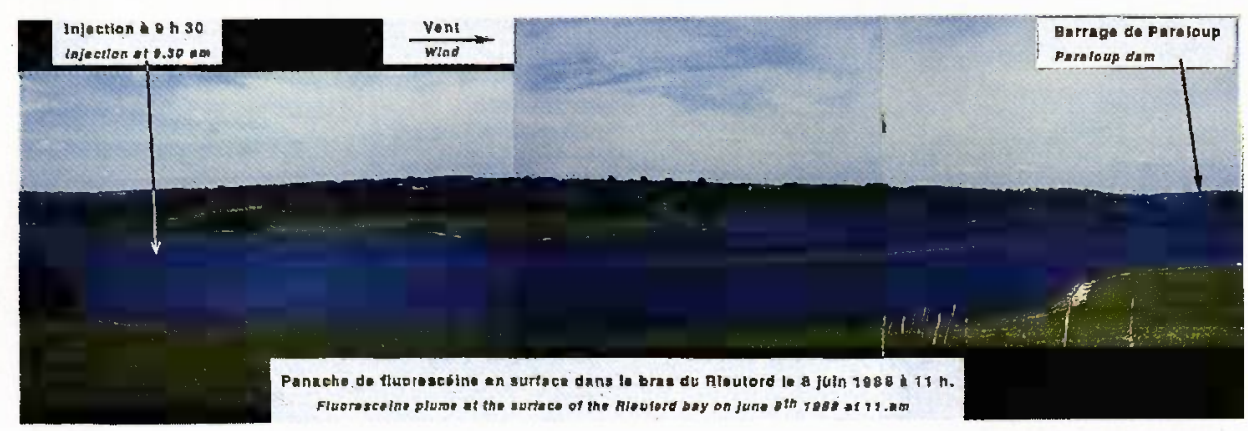

Fig. 15. - Panache de fluorescéine en surface dans le bras du Rieutord le 8 juin 1988.

Fig. 15. - Fluorescein plume on the surface of the arm of the Rieutord. June 8, 1988. 
ling s'est établi dans le fond du bras, avec apparition en surface de l'eau de l'hypolimnion marquée à la rhodamine. Les eaux de surface, marquée à la fluorescéine, ont été très rapidement entraînées par le vent vers le centre du lac avec une vitesse maximale comprise entre $370 \mathrm{~m} \cdot \mathrm{h}^{-1}$ en rive droite du bras et $310 \mathrm{~m} \cdot \mathrm{h}^{-1}$ dans la retenue et se sont très vite dispersées (fig. 15).

Le deuxième marquage a été réalisé le 16 septembre 1988 par fort vent de Nord-Ouest, établi depuis 4 jours (fig. 17) et en présence d'une stratification marquée. Ce marquage a permis de mettre en évidence des circulations dans l'épilimnion par boucles verticales (Calmels et al., 1989b). L'injection de fluorescéine réalisée à $-13 \mathrm{~m}$, juste au-dessus de la thermocline, a cheminé en sens contraire du vent (vers le Nord) et cette circulation a été déviée par le haut-fond qui relie l'île de Puech à la presqu'île de Charouzech. Le traceur est alors remonté en surface dans une zone d'émergences de bouffées d'eau marquée. Cette zone était localisée au droit des isobathes $-12 \mathrm{~m}$ à $-14 \mathrm{~m}$. Le traceur a été ensuite entraîné dans la circulation de surface vers le Sud. Les eaux de surface (jusqu'à une profondeur de $-8 \mathrm{~m}$ ) sont entraînées dans le sens du vent, vers le Sud avec une vitesse de l'ordre de $100 \mathrm{~m} \cdot \mathrm{h}^{-1}$. Les eaux situées au-dessus de la thermocline (entre -8 et $-13 \mathrm{~m}$ ) sont entraînées dans le sens inverse du vent avec une vitesse de $200 \mathrm{~m} \cdot \mathrm{h}^{-1}$ (fig. 16).
Les marquages réalisés à la rhodamine dans l'hypolimnion ont mis en évidence un échappement de l'eau du fond par le thalweg étroit qui contourne l'ile du Puech par l'Ouest. La vitesse maximale mesurée à $-18 \mathrm{~m}$ était de $180 \mathrm{~m} \cdot \mathrm{h}^{-1}$.

\section{IV.4 Échanges des masses d'eau dans le détroit de Charouzech}

Le détroit de Charouzech correspond à un rétrécissement en gorge de la vallée du Vioulou, avec un dénivelé de quelques mètres sur une courte distance, et un coude à angle droit.

Lorsque la retenue est pleine, la largeur en surface du détroit $(\approx 400$ mètres) ne permet pas de soupçonner les phénomènes hydrodynamiques dont il est le siège lorsque soufflent les vents dominants (de Nord-Ouest principalement et de Sud-Est moins fréquemment).

Les marquages réalisés en septembre 1988 (Calmels et al., 1989b), puis en juin et septembre 1989 (Calmels et al., 1990a; 1990b), dans des conditions de vent différentes, ont progressivement mis en évidence le rôle fondamental que joue ce détroit sur les échanges hydrauliques entre les parties Est et Ouest du lac, du fait du rétrécissement de la section d'échange, de l'angle du sillon et du dénivelé. 

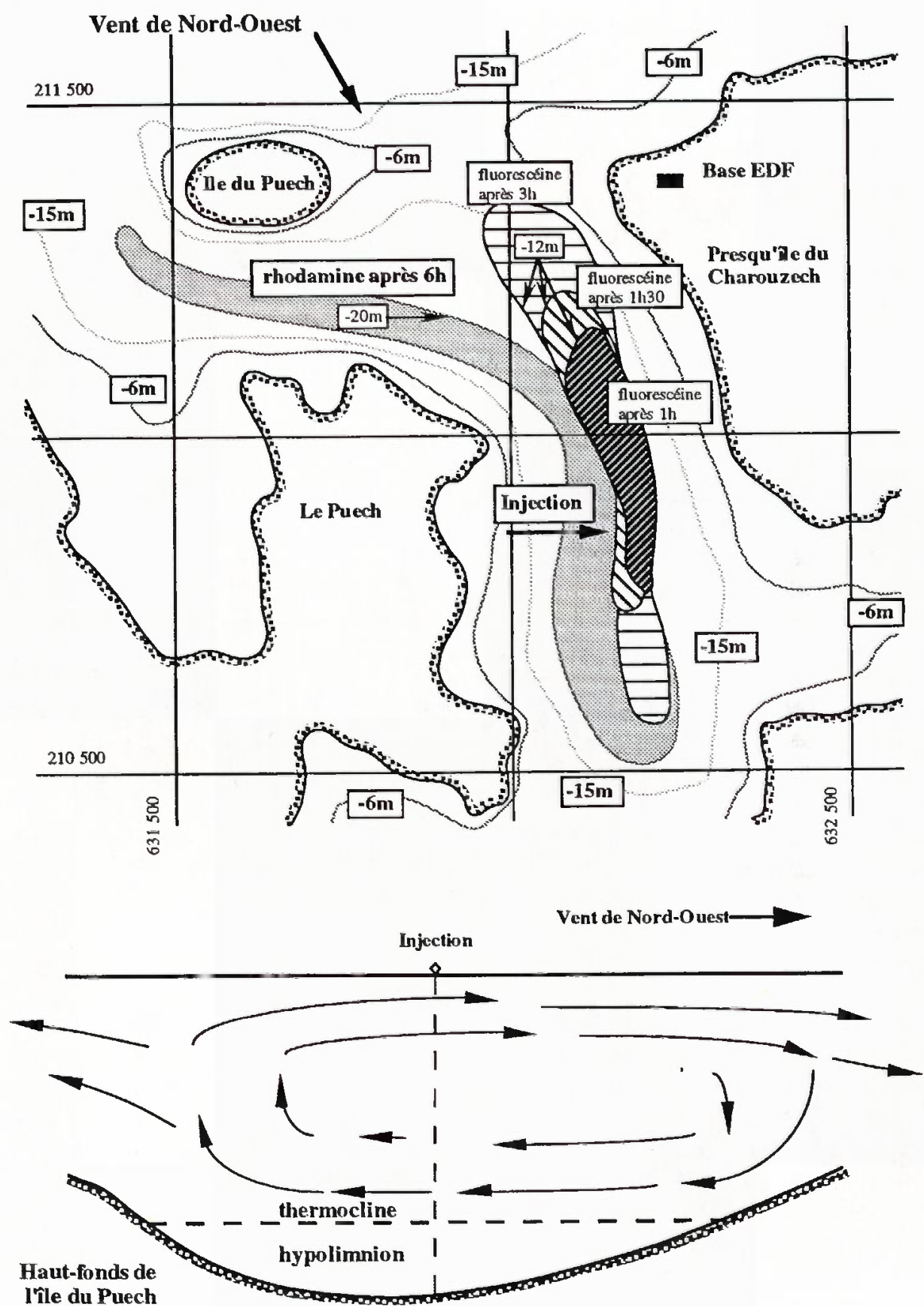

Fig. 16. - Évolution des masses d'eau marquées et schéma des écoulements dans le bras du Rieutord par vent de Nord-Ouest.

Fig. 16. - Movement of marked water masses and diagram of flows in the arm of the Rieutord with a north-westerly wind. 

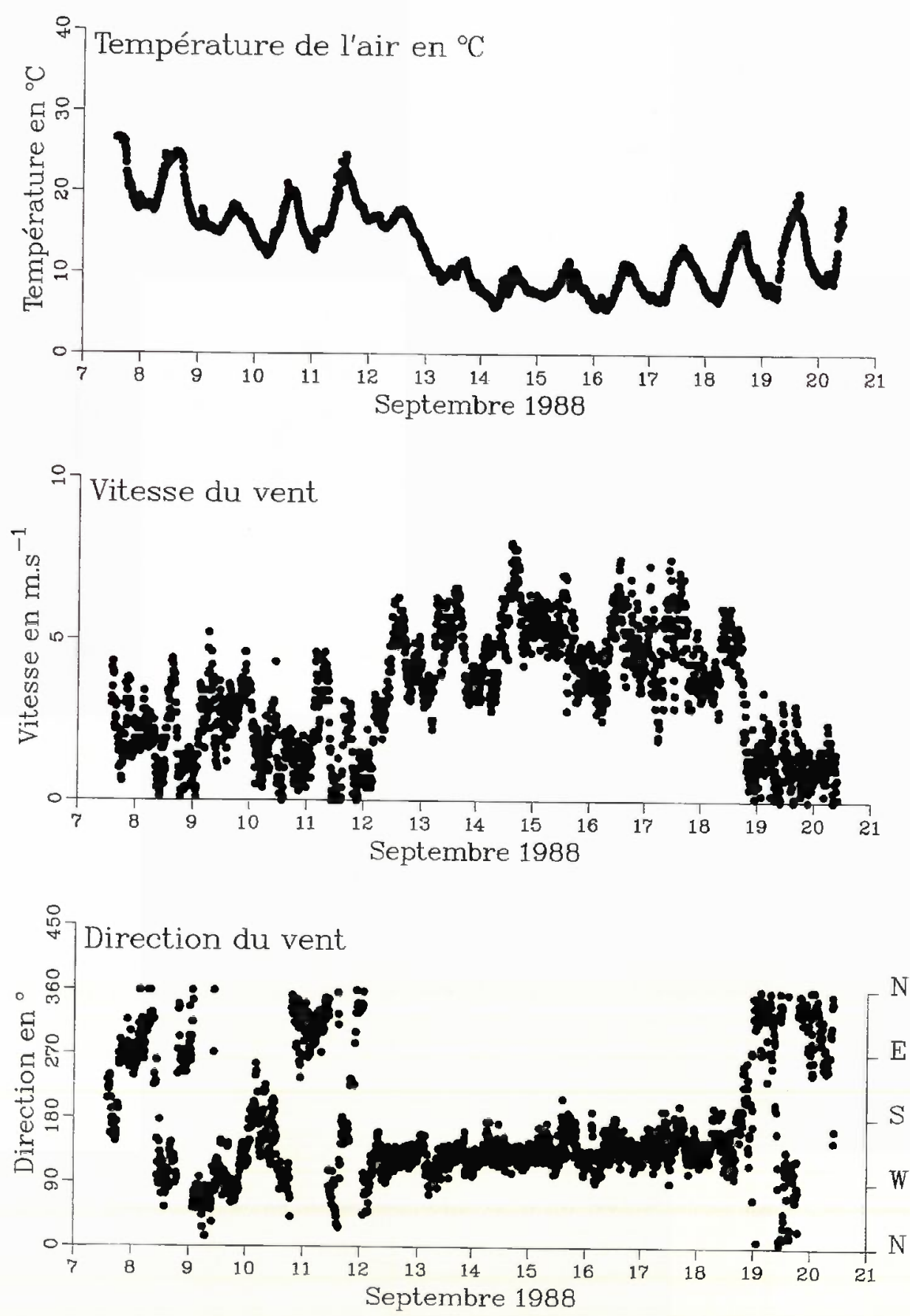

Fig. 17. - Température de l'air, vitesse et direction du vent mesurés sur la plateforme. Septembre 1988. Fig. 17. - Air temperature, speed and direction of wind measured from the platform. September 1988. 


\section{IV.4.1 Marquages par vent de Nord-Nord-Ouest}

Deux marquages ont été réalisés par vent de Nord-Nord-Ouest.

A l'époque de l'année où la stratification est la plus forte, la campagne de septembre 1988 a eu lieu pendant que soufflait un fort vent établi de Nord-Ouest (5 à $7 \mathrm{~m} \cdot \mathrm{s}^{-1}$ ) (fig. 17), ce qui a permis de mettre en évidence un régime permanent de circulation des masses d'eau. Cette situation était associée à un fort refroidissement climatique, qui a entraîné une forte déstratification (fig. 18). La thermocline étant située, au début du marquage, entre $-12 \mathrm{~m}$ et $-16 \mathrm{~m}$, une injection de fluorescéine a été réalisée de 0 à $-12 \mathrm{~m}$, et une injection de rhodamine de -16 à $-24 \mathrm{~m}$.
Ces marquages différenciés en surface et au fond ont permis de mettre en évidence une circulation d'eau par boucles verticales. Au niveau du rétrécissement, deux boucles prennent naissance, une dans l'épilimnion, l'autre dans l'hypolimnion, induites par la rupture de géométrie de la vallée.

En surface, de 0 à $-10 \mathrm{~m}$ l'eau s'écoule dans le sens du vent (vitesse maximale en surface $114 \mathrm{~m} \cdot \mathrm{h}^{-1}$ ), alors qu'un courant inverse alimente la boucle de l'épilimnion, juste au-dessus de la thermocline, de -10 à $-14 \mathrm{~m}$ (vitesse maximale $80 \mathrm{~m} \cdot \mathrm{h}^{-1}$ ) (fig. 19). La limite de la boucle de surface, matérialisée par une zone d'émergences de bouffées d'eau marquée, est située au niveau du fort rétrécissement bathymétrique du détroit du Charouzech. Le courant inverse horizontal circulant

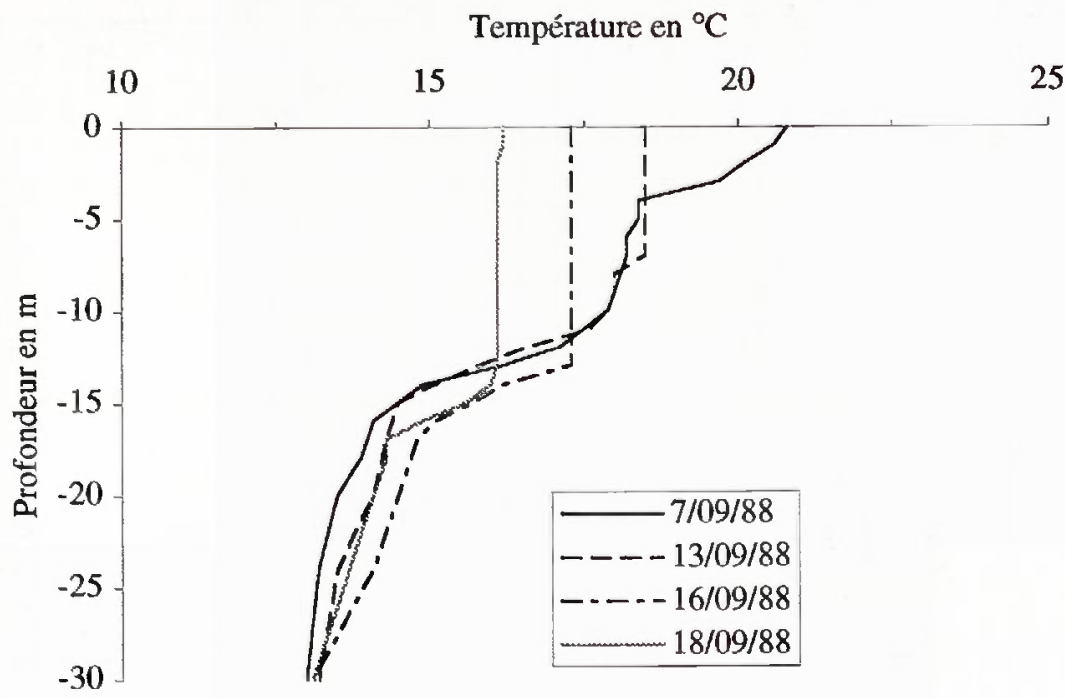

Fig. 18. - Profils thermiques à la plateforme en septembre 1988 .

Fig. 18. - Thermal profiles at the platform in September 1988. 


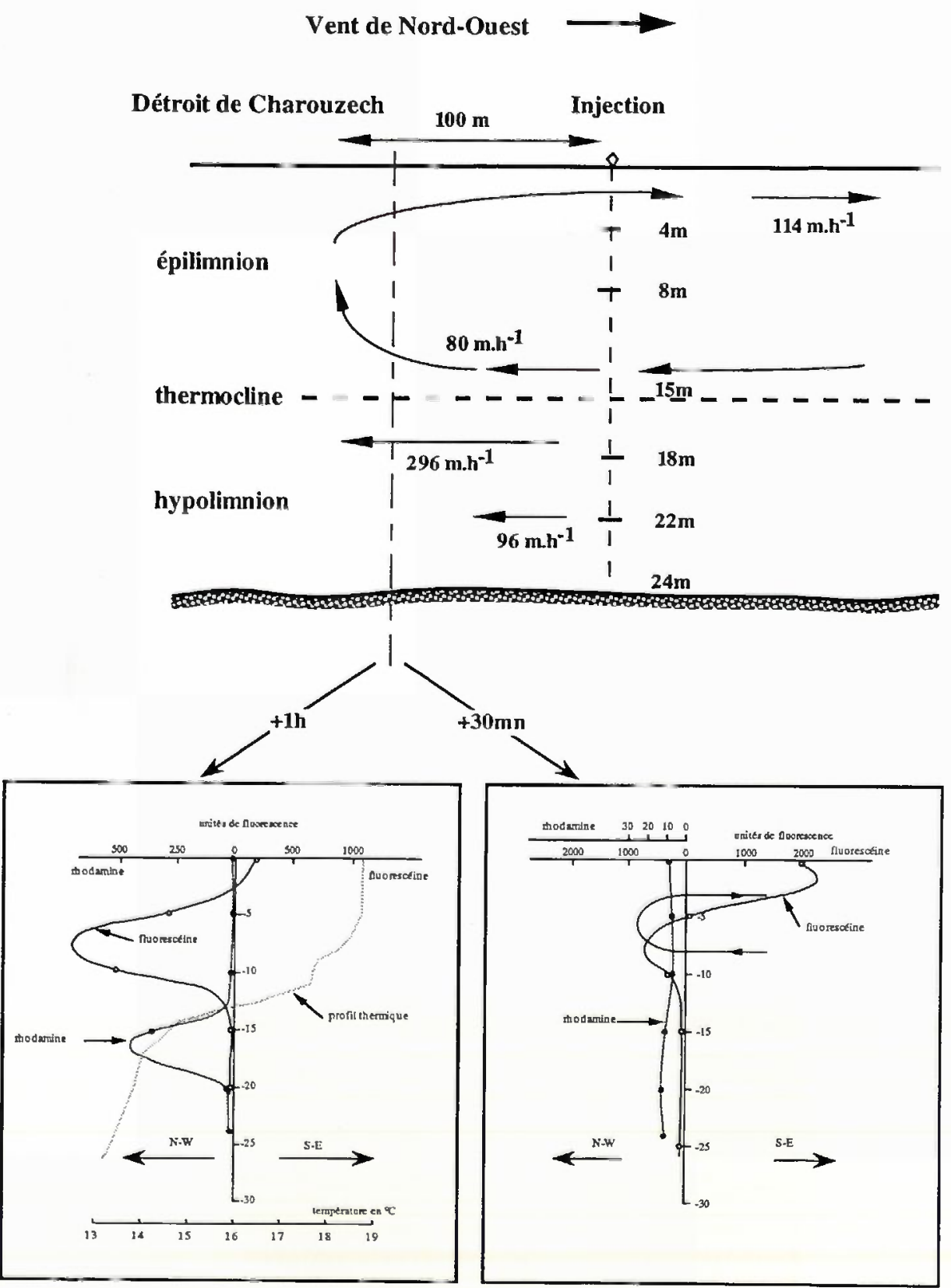

Fig. 19. - Schématisation des courants mesurés dans le détroit de Charouzech.

Fig. 19. - Schematic view of currents measured in the Charouzech strait. 
vers $-12 \mathrm{~m}$ est alors dévié verticalement et parvient ainsi en surface où il rejoint la circulation dans le sens du vent (fig. 20).

Une circulation similaire s'établit dans l'hypolimnion juste au-dessous de la thermocline. A ce niveau-là, le détroit est un véritable goulet. Les masses d'eau marqués à la rhodamine ont été entraînées vers la plateforme (fig. 20). II n'a été possible de mesurer que la vitesse orientée vers l'Ouest (vitesse maximale de
$269 \mathrm{~m} \cdot \mathrm{h}^{-1}$ à $-18 \mathrm{~m}$ et $96 \mathrm{~m} \cdot \mathrm{h}^{-1}$ à $-22 \mathrm{~m}$ ), ce qui n'a pas permis de mettre en évidence la présence d'une boucle de courant, symétrique de celle qui existe dans l'épilimnion.

Les valeurs du nombre de Wedderburn à cette période $(3<W<10)$ (Salençon, 1994) confirment que l'automne présente des conditions favorables pour une forte production de turbulence, au niveau de la thermocline, par le cisaillement dû à ces circulations par boucles verticales. C'est

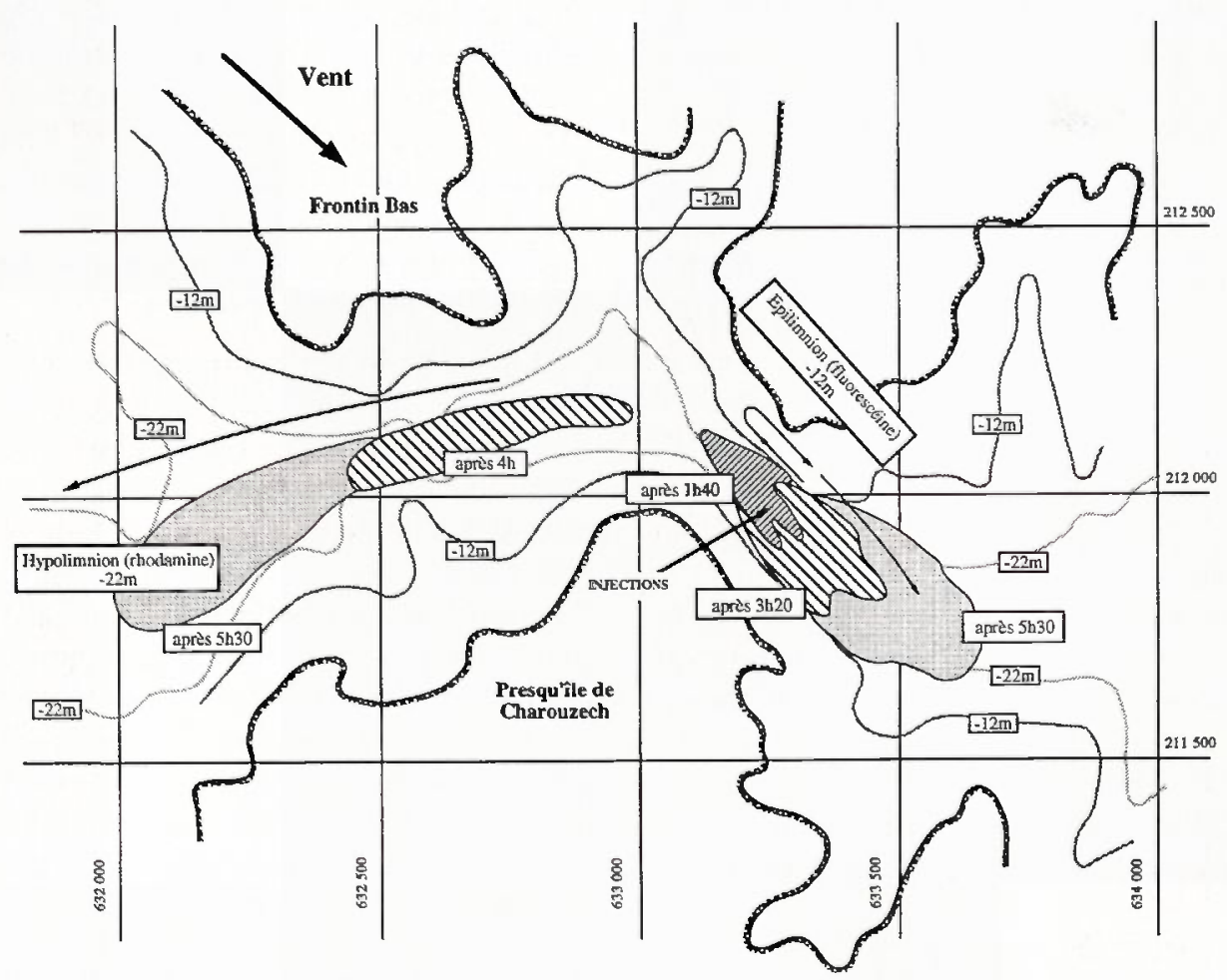

Fig. 20. - Évolution et situations des masses d'eau marquées le 14 septembre 1988 dans le détroit de Charouzech.

Fig. 20. - Movement and location of marked water masses on September 14, 1988 in the Charouzech strait. 


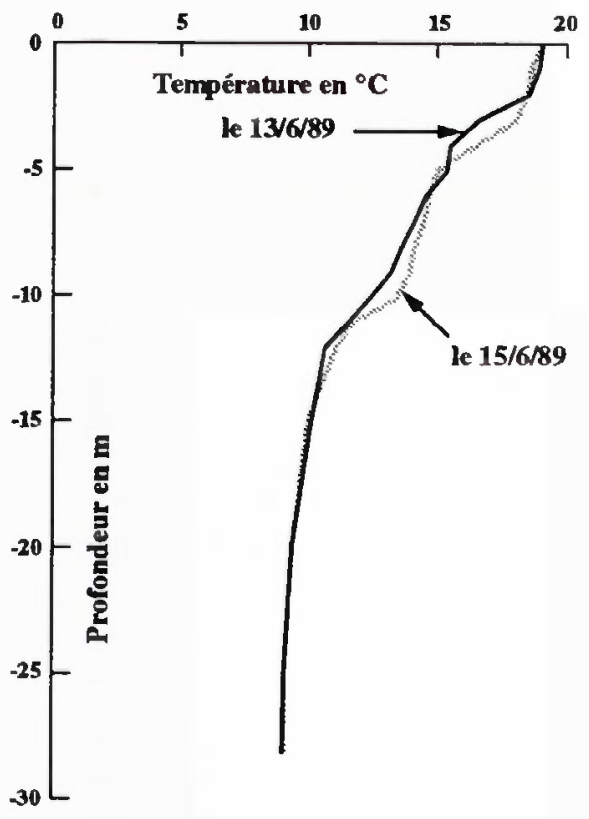

Fig. 21. - Profils thermiques dans le détroit de Charouzech en juin 1989.

Fig. 21. - Thermal profiles in the Charouzech strait in June 1988.

un facteur très important dans l'étape de déstratification automnale.

En juin 1989, les marquages ont été influencés par la présence d'une thermocline transitoire, située entre $-4 \mathrm{~m}$ et $-6 \mathrm{~m}$, qui résulte d'une période chaude et peu ventée précédant la campagne. La thermocline saisonnière étant située à $-10 \mathrm{~m}$ (fig. 21 ), cette thermocline transitoire a joué un rôle d'écran au mélange vertical.

Pendant toute la durée des marquages, le vent est resté faible $\left(3 \mathrm{~m} \cdot \mathrm{s}^{-1}\right)$ orienté au Nord-Nord-Ouest et tournant à l'Est en s'affaiblissant en fin de journée.
Une injection de fluorescéine a été réalisée à $-5 \mathrm{~m}$ au milieu de l'entrée Est du détroit. Quelques minutes après le début de l'injection, des volutes de remontée de la fluorescéine vers la surface ont été observées dans une zone d'émergence, située au Nord-Est du point d'injection. Le panache de surface ainsi formé s'est ensuite étiré en surface vers SallesCuran, dans la direction du vent, avec une vitesse de $440 \mathrm{~m} \cdot \mathrm{h}^{-1}$, a conservé une alimentation par la zone d'émergence pendant les $3 \mathrm{~h}$ qui ont suivi l'injection (fig. 22).

Une injection de rhodamine a été effectuée vers $-9 \mathrm{~m}$, dans la couche de l'épilimnion comprise entre la thermocline saisonnière et la thermocline transitoire. Cette injection n'a été suivi d'aucune remontée du traceur en surface, ce qui a confirmé le rôle d'écran de la thermocline transitoire.

Le panache est resté confiné entre les deux thermoclines, amorçant un mouvement en direction du détroit de Charouzech mais sans pouvoir franchir la limite précédemment détectée, qui semble représenter une frontière hydraulique. Le panache s'est ensuite dirigé vers la partie Est dans un mouvement giratoire à la fois horizontal et vertical. Une cartographie réalisée 15 heures après l'injection a montré que le traceur a envahi toute la partie Est de la retenue, en restant à la profondeur $-9 \mathrm{~m}$ (fig. 23).

\section{IV.4.2 Marquages par vent de Sud}

Les marquages des 7 et 8 septembre 1989 ont été réalisés alors qu'un vent 


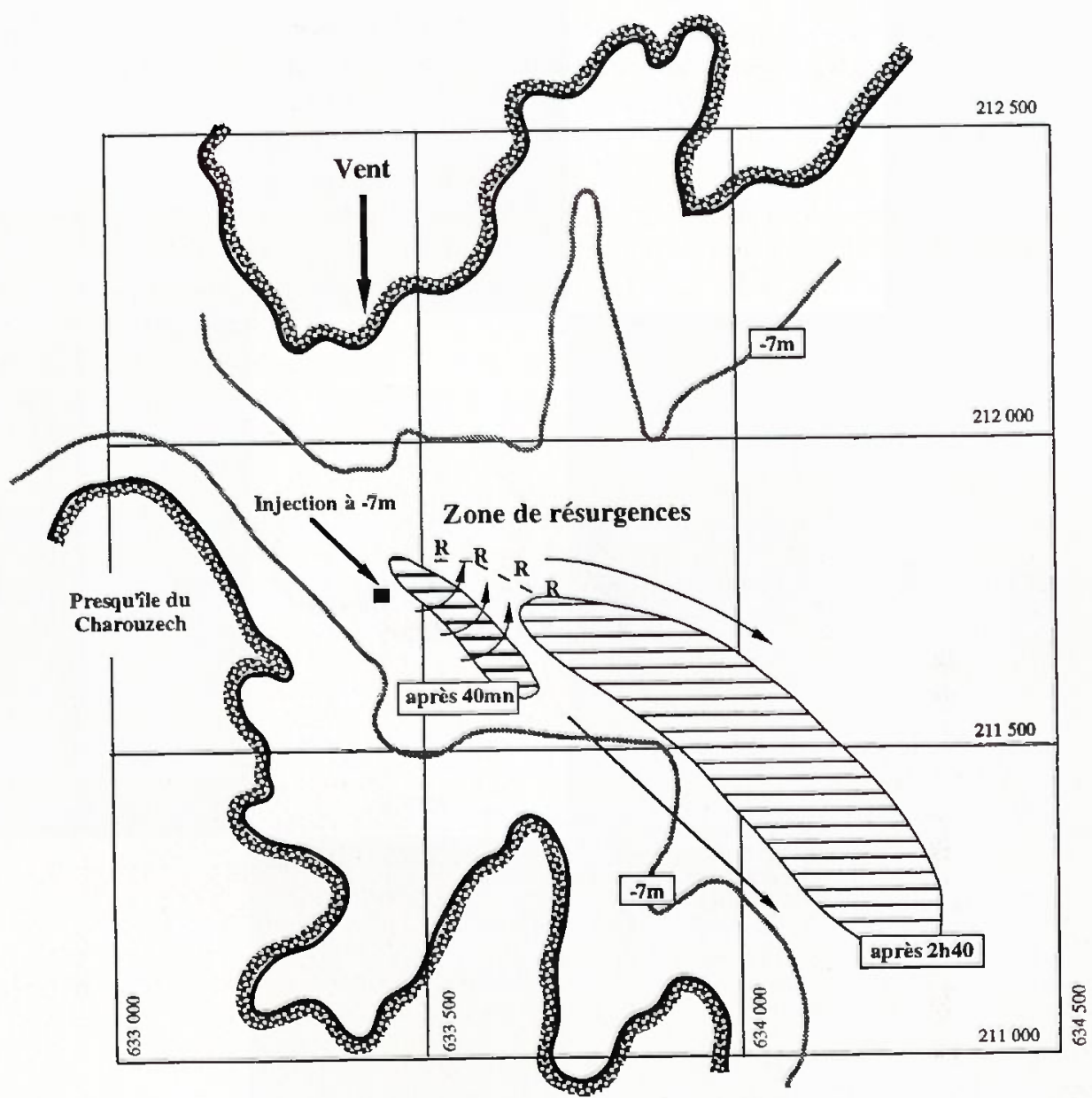

Fig. 22. - Évolution du panache de fluorescéine en surface dans le détroit du Charouzech le 14 juin 1989.

Fig. 22. - Movement of the fluorescein plume at the surface in the Charouzech strait on June 14, 1989.

de Sud était établi depuis deux jours (fig. 24), la thermocline étant située vers $-12 \mathrm{~m}$.

Une injection de fluorescéine dans l'épilimnion a montré qu'une circulation en sens inverse du vent située à $-10 \mathrm{~m}$, progressait vers la rive Sud avec une vitesse de $50 \mathrm{~m} \cdot \mathrm{h}^{-1}$. Après avoir rencontré l'isobathe $-10 \mathrm{~m}$ les courants ont été déviés vers la surface en créant une zone d'émergence du traceur (fig. 25). En surface, le traceur a progressé dans le sens du vent avec une vitesse de $240 \mathrm{~m} \cdot \mathrm{h}^{-1}$ en sur- 
face et $165 \mathrm{~m} \cdot \mathrm{h}^{-1}$ à $-5 \mathrm{~m}$, tout en étant alimenté par les émergences pendant plusieurs heures (4 heures observées).

Pour étudier les circulations dont l'hypolimnion est le siège par vent de Sud-Est établi, une injection ponctuelle de rhodamine a été réalisée à
$-20 \mathrm{~m}$ au milieu de l'entrée Est du détroit, le 8 septembre 1989.

Dans un premier temps, un panache très allongé s'est formé et s'est déplacé vers le Nord-Ouest, conformément aux circulations observées auparavant à $-20 \mathrm{~m}$, avec une vitesse maximale de $170 \mathrm{~m} \cdot \mathrm{h}^{-1}$ (fig. 26).

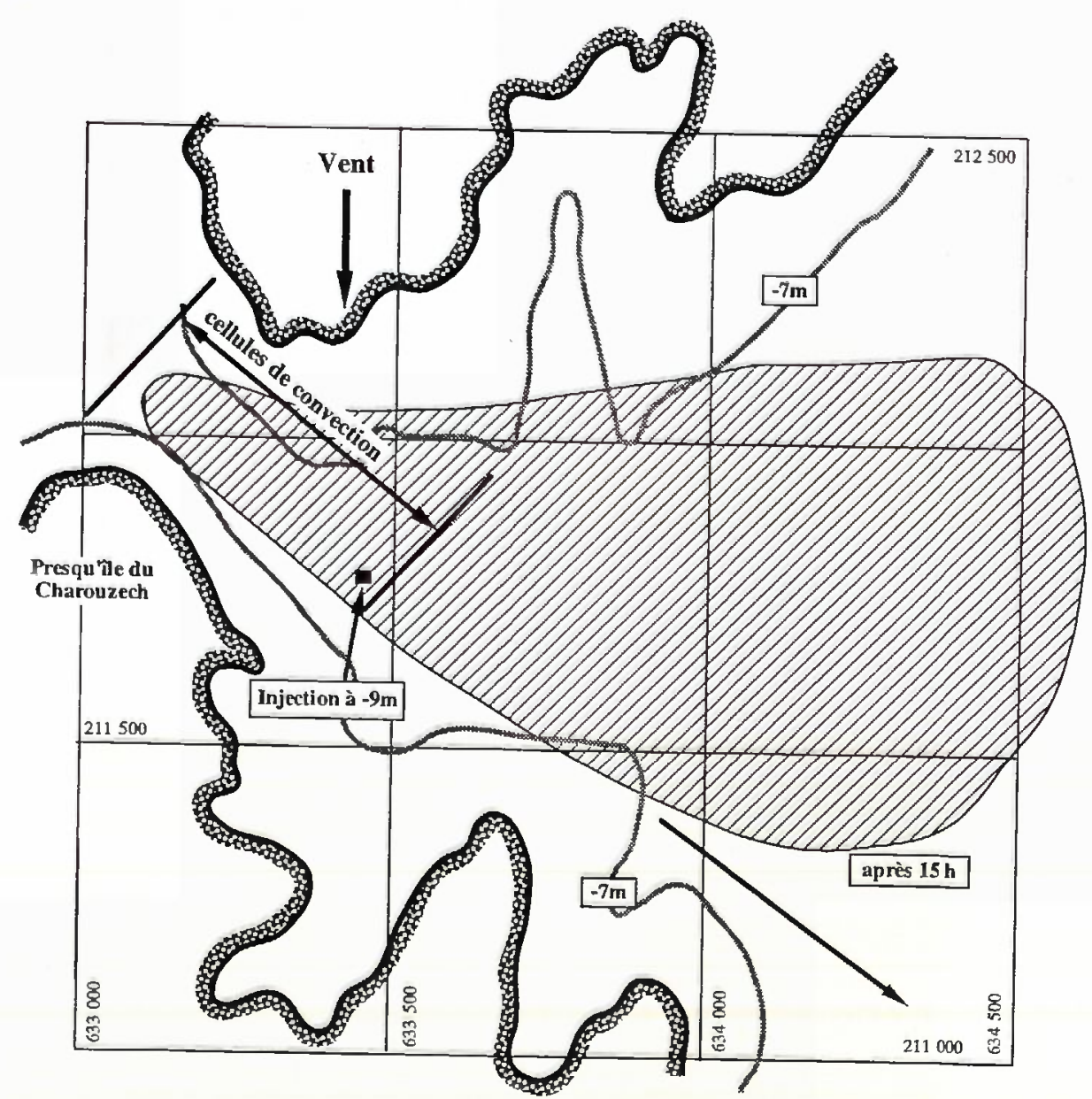

Fig. 23. - Cartographie du panache de rhodamine à $-9 \mathrm{~m}$, 15 heures après l'injection, le 16 juin 1989.

Fig. 23. - Cartography of the rhodamine plume at $-9 \mathrm{~m}, 15$ hours after injection, June $16,1989$. 

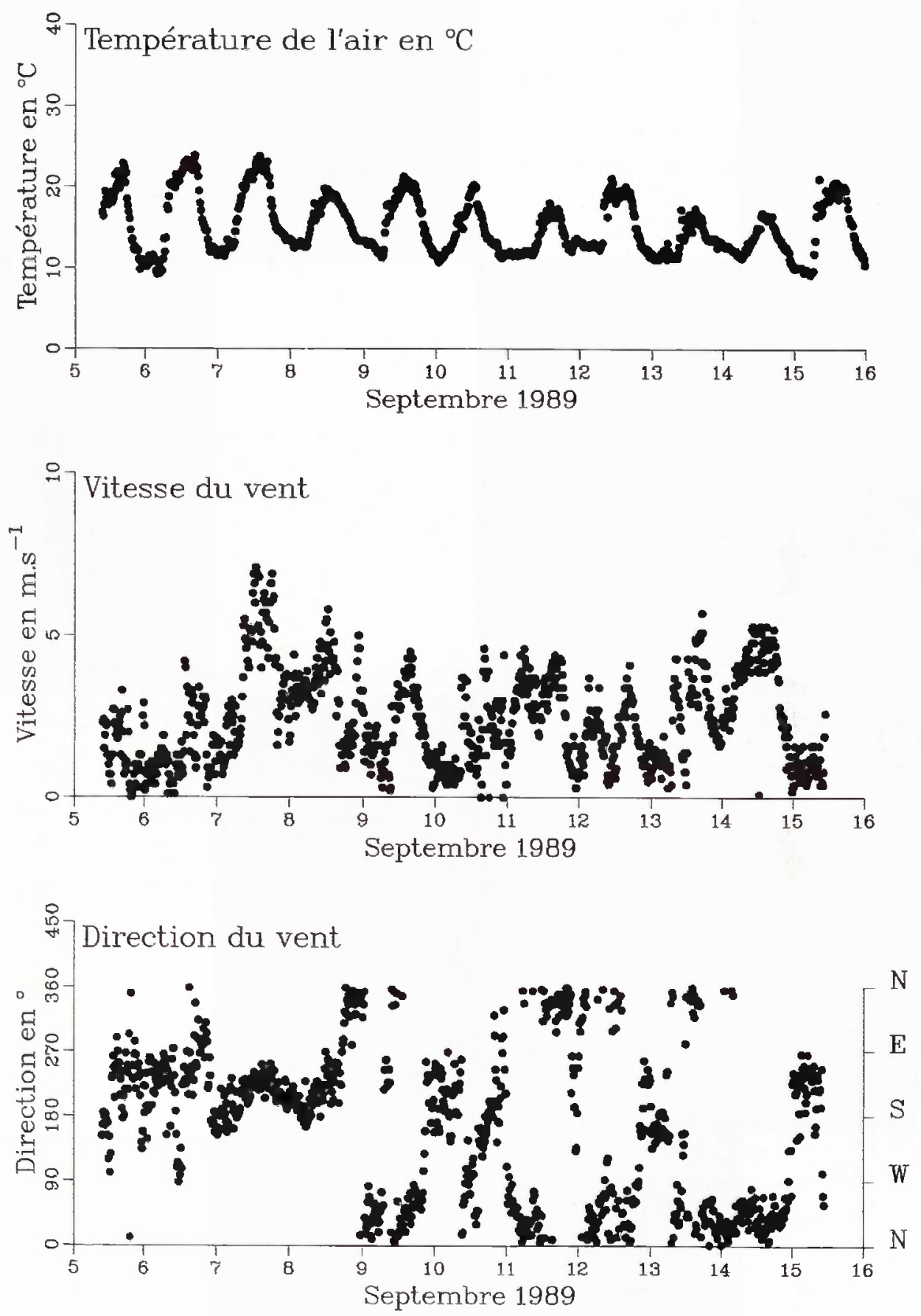

Fig. 24. - Température de l'air, vitesse et direction du vent mesurés sur la plateforme. Septembre 1989. Fig. 24. - Air temperature, speed and direction of wind measured from the platform. September 1989. 
Ensuite, le panache a continué à envahir progressivement le thalweg du Vioulou en direction du barrage en se dispersant entre la thermocline $(-15 \mathrm{~m})$ et une cote située vers $-25 \mathrm{~m}$, sans atteindre le fond.

Une cartographie, réalisée 24 heures après l'injection alors que le vent était tombé, a révélé que le panache a progressé jusqu'à la plateforme, le front amont restant situé dans le détroit de Charouzech.

Une autre cartographie, réalisée 6 jours après l'injection alors que le vent a été orienté au Nord-Nord-Ouest, a montré que le panache avait atteint

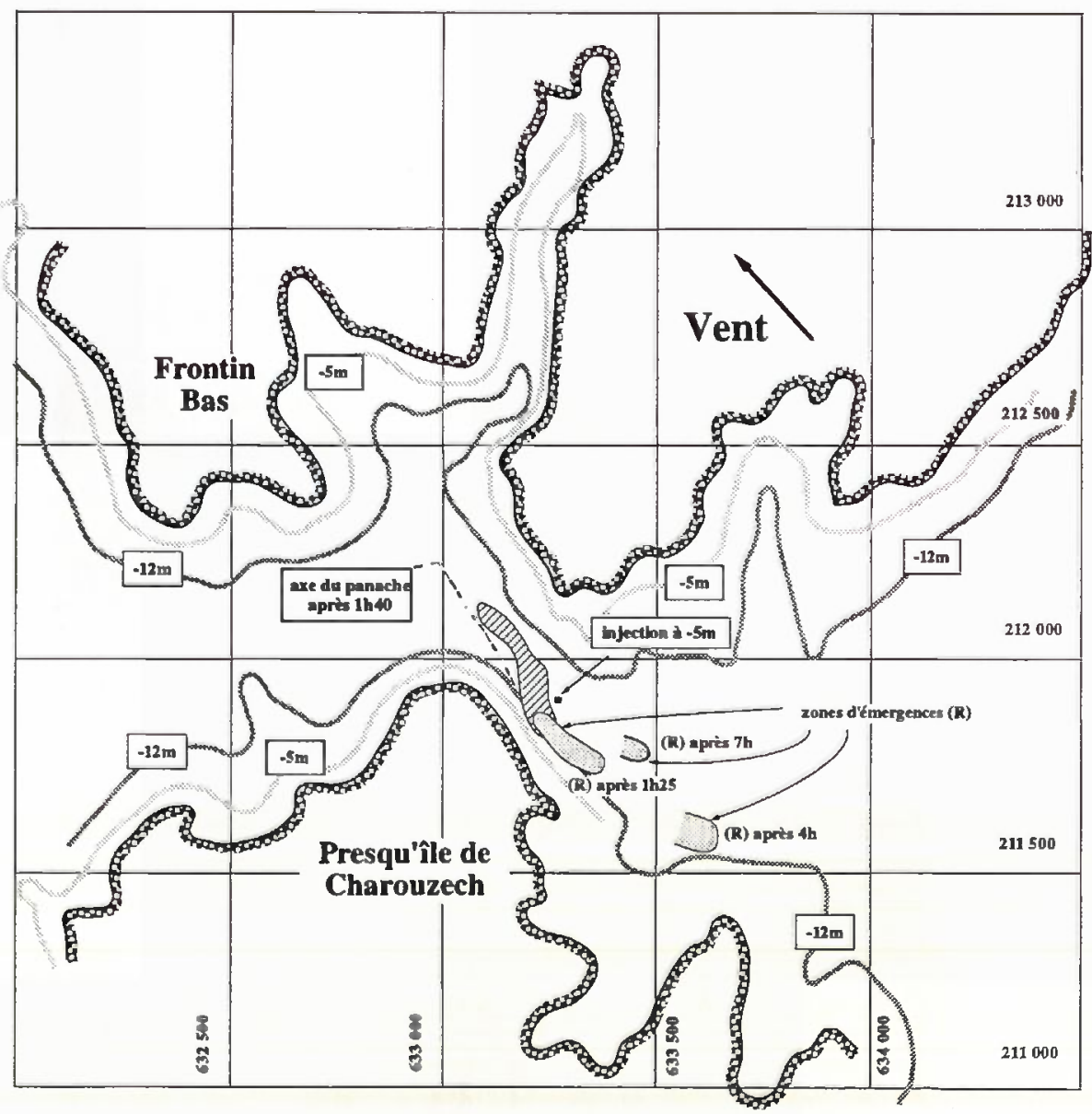

Fig. 25. - Dispersion du panache de surface par vent de Sud dans le détroit de Charouzech le 7 septembre 1989.

Fig. 25. - Dispersion of the surface plume by the south wind in the Charouzech strait on September 7 , 1989. 


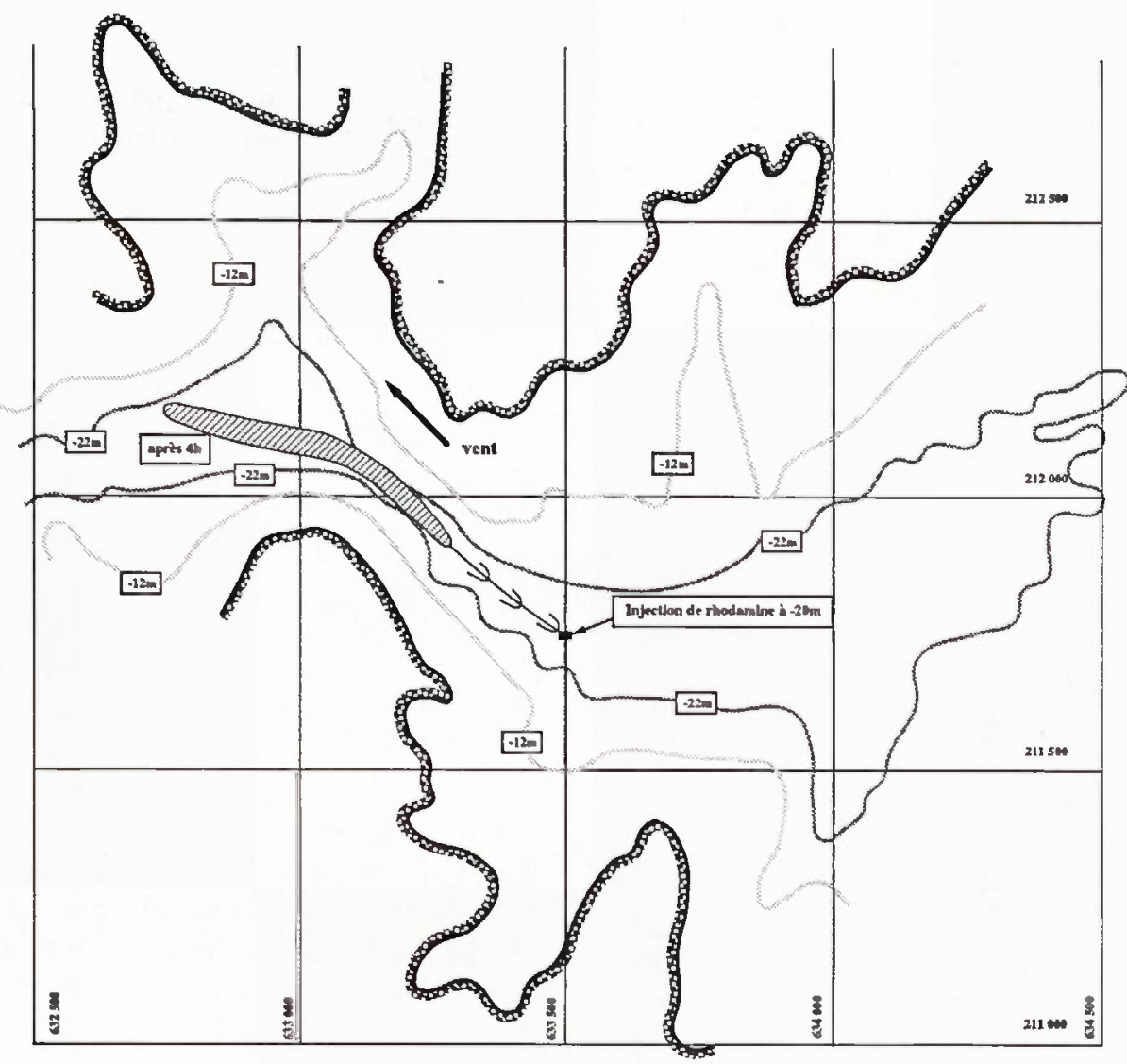

Fig. 26. - Evolution du panache de rhodamine à $-20 \mathrm{~m}$ dans le détroit de Charouzech par vent de Sud.

Fig. 26. - Movement of the rhodamine plume at $-20 \mathrm{~m}$ in the Charouzech strait with a south wind.

le barrage. Le front amont était toujours situé au centre du détroit et $y$ est resté fixe durant les 6 jours qu'ont duré les observations, sans jamais repartir vers la partie Est de la retenue (fig. 27).

Cette occupation progressive de toute la partie Ouest de la retenue (entre le barrage et le détroit) est similaire à celle qui avait été observée lors de l'étude du panache de rejet du pompage de stockage.

Le détroit de Charouzech semble bien représenter une frontière pour la circulation par vent du Nord. 


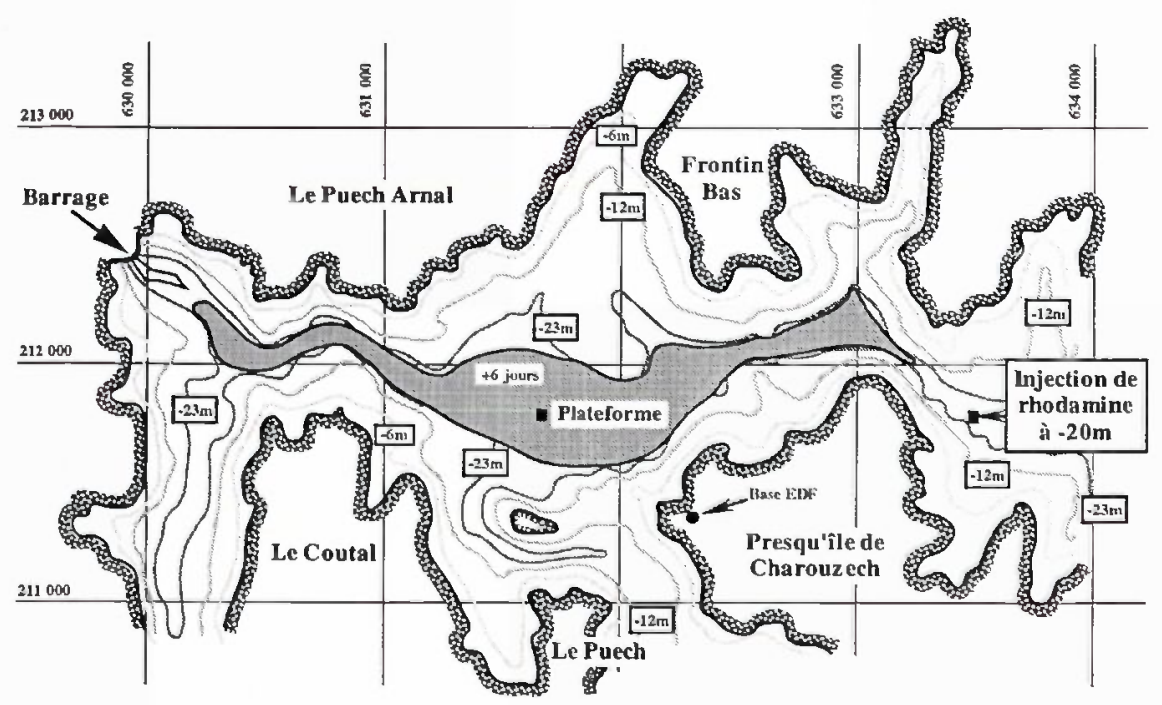

Fig. 27. - Cartographie du panache de rhodamine à $-20 \mathrm{~m}, 6$ jours après l'injection.

Fig. 27. - Cartography of the rhodamine plume at $-20 \mathrm{~m}, 6$ days after injection.

\section{IV.4.3 Conclusion}

Le détroit de Charouzech semble se comporter comme une frontière hydraulique, en particulier dans I'hypolimnion, freinant les échanges entre les parties Est et Ouest de la retenue. Cependant, ces effets sont marqués surtout lors de situations de vent bien établies. Lorsque l'orientation du vent fluctue, nous avons mis en évidence une rapide homogénéisation horizontale.

En effet, chaque campagne réalisée en septembre à la suite d'une campagne en juin a été précédée par une cartographie de traceur résiduel. Cette cartographie a montré que la rhodamine qui avait été injectée en juin dans l'hypolimnion n'était présente que dans l'hypolimnion en septembre et qu'elle était répartie uniformément dans toute l'étendue de la retenue, y compris jusque dans les baies de la partie Est. Ces résultats montrent l'activité des courants de densité qui se chargent en permanence de rétablir l'homogénéité horizontale par advection.

Les phénomènes que nous avons mis en évidence sont donc importants en tant que «moteurs locaux» car ils peuvent relancer très rapidement une activité biologique en situation de carence (par l'apport de nutriments en surface grâce aux boucles verticales par exemple). En effet, l'observation de cette retenue à une échelle de temps et d'espace plus grande ne révèle qu'une étendue d'eau stratifiée homogène horizontalement et ne permet pas de soupçonner un tel potentiel de réaction, capable d'influencer considérablement la dynamique de l'écosystème. 


\section{v CONCLUSION}

La circulation des masses d'eau de Pareloup a été étudiée au cours de cinq campagnes de marquage avec des traceurs fluorescents.

Ces campagnes ont permis de mettre en évidence certaines circulations complexes qui s'établissent en réponse aux sollicitations extérieures (climatiques et hydrauliques).

Le suivi du panache de l'eau pompée à Bage a montré que, dès qu'il existe une légère stratification, l'eau de Bage reste confinée dans l'hypolimnion. Le panache progresse horizontalement à son niveau de densité, envahissant progressivement les thalwegs latéraux jusqu'au détroit de Charouzech. Si ce panache ne détruit pas la stratification de la retenue, il est susceptible de remonter en surface lors d'un upwelling, essentiellement au printemps. Ainsi, les eaux riches du Viaur peuvent participer à la production phytoplanctonique de Pareloup à des périodes où le bloom printanier de diatomées a épuisé le stock hivernal de nutriments.

Les échanges de masses d'eau de part et d'autre du détroit de Charouzech sont complexes. Le fort rétrécissement bathymétrique associé à un coude du thalweg fait jouer à ce détroit un rôle de barrière hydraulique pour les déplacements d'eau de l'Ouest vers l'Est, lorsque le vent est de secteur Nord, en particulier dans l'hypolimnion.
Les circulations par boucles verticales qui s'établissent rapidement lors d'un coup de vent en période stratifiée permettent une remontée en surface des eaux du métalimnion, plus riches en nutriment.

La rapidité de la réponse de ce lac montre que la dynamique de son écosystème, dont nous observons les résultats au mieux à une échelle journalière ou hebdomadaire s'appuie sur des phénomènes rapides et locaux dont nous avons beaucoup de difficultés à estimer le rôle et l'ampleur. Comprendre ces phénomènes transitoires est une clé importante pour la compréhension de la dynamique d'un écosystème.

\section{REMERCIEMENTS}

Nous tenons à remercier tous les membres de l'équipe "traçages" pour leur collaboration à ces campagnes, en particulier P. Mouyon pour son efficacité sur le terrain et son intarissable bonne humeur, $\mathrm{H}$. Pujo qui a assuré en "bougonnant" toute la localisation au théodolite, et J.M. Nayral, chargé de la navigation, qui nous a fourni une aide précieuse en nous faisant bénéficier de la connaissance du terrain qu'il a acquise "depuis tout-petit " en grandissant sur les bords du lac. Merci de nous avoir soutenus pendant les heures noires où le traceur avait sombré dans un trou noir! 


\section{RÉFÉRENCES BIBLIOGRAPHIQUES}

Calmels P. et Salençon M.J., 1991. Techniques de marquage pour l'étude des circulations des masses d'eau dans les lacs. Application à la retenue de Pareloup. In : IAEA (Editor), Use of Isotope Techniques in Water Resources Development. Proc. Symp. 11-15 March 1991, Vienna. IAEA/UNESCO, Vienna, pp. 107-122.

Calmels P., Marion D. et Salençon M.J., 1989a. Traçage des masses d'eau dans la retenue de Pareloup. Marquage continu du pompage. Effet du soutirage. Echanges dans le bras du Rieutord - Juin 1988. Rapport HE31/89-2, Electricité de France, $\mathrm{Pa}$ ris, $24 \mathrm{pp}$.

Calmels P., Mouyon P. et Salençon M.J., 1989b. Traçage des masses d'eau dans la retenue de Pareloup. Marquage des eaux dans le détroit du Charouzech et dans le bras du Rieutord - Septembre 1988. Rapport HE31/89-3, Electricité de France, $\mathrm{Pa}$ ris, $38 \mathrm{pp}$.

Calmels P., Mouyon P. et Salençon M.J., 1990a. Traçage des masses d'eau dans la retenue de Pareloup. Marquage des eaux dans le détroit du Charouzech et dans le bras du Rieutord - Juin 1989. Rapport HE31/90-12, Electricité de France, Paris, 36 pp.

Calmels P., Mouyon P. et Salençon M.J., 1990b. Traçage des masses d'eau dans la retenue de Pareloup. Marquage des eaux dans le détroit du Charouzech et le détroit d'Arnal - Sep- tembre 1989. Rapport HE31/90-15, Electricité de France, Paris, $26 \mathrm{pp}$.

Gilath Ch. and Gonfiantini R., 1983. Lake dynamics, Guidebook on nuclear techniques in hydrology, Technical reports series $n^{\circ} 91$. AIEA Edition, Vienna, pp. 129-157.

Imberger J., 1979. Mixing in reservoirs. In : H.G. Fischer, E.J. List, R.C.Y. Koh, J. Imberger and N.H. Brooks (Editors), Mixing in Inland and Coastal Waters. Academic Press, New York, pp. 148228.

Imberger J., 1987. Hydrodynamics of lakes. In : Australian Water and Wastewater Assoc. (Editor). $12^{\text {th }}$ Convention Proc. Symp. AWWA, Adelaïde, pp. 401-423.

Imberger J. and Monismith S.G., 1986. Appendix to: Monismith, S.G., 1986. An experimental study of the upwelling response of stratified reservoirs to surface shear stress. J. Fluid Mech., 171: 407-439.

Patterson J.C., Hamblin P.F. and Imberger J., 1984. Classification and dynamic simulation of the vertical density structure of lakes. Limnol. Oceanogr., 29 : 845-861.

Pujo H., 1994. Levé bathymétrique du réservoir de Pareloup. Hydroécol. Appl. 6(1/2), pp.

Salençon M.J., Pujo H., Calmels P. et Marion D., 1988. Traçage des masses d'eau de la retenue de Pareloup : septembre 1987. Rapport HE31/88-15, Electricité de France, Paris, 28 pp.

Salençon M.J., 1994. Stratification thermique d'un réservoir: le modèle à bilan d'énergie, EOLE. Rapport HE31/94-1, Electricité de France, Paris, $113 \mathrm{pp}$. 